\title{
1 Antimicrobials from a feline commensal bacterium inhibit skin infection by
}

\section{drug-resistant $S$. pseudintermedius.}

4

5 Authors: Alan M. O’Neill ${ }^{1}$, Kate A. Worthing ${ }^{2}$, Nikhil Kulkarni ${ }^{1}$, Fengwu Li ${ }^{1}$, Teruaki

6 Nakatsuji ${ }^{1}$, Robert H. Mills ${ }^{3,4}$, Joyce Y. Cheng ${ }^{1}$, David J. Gonzalez ${ }^{3,4}$, Jacqueline M. Norris ${ }^{5}$

$7 \quad$ and Richard L. Gallo ${ }^{1 *}$

8

9 Affiliations:

$10{ }^{1}$ Department of Dermatology, University of California, San Diego, CA, USA.

$11{ }^{2}$ College of Veterinary Medicine, University of Arizona, Tucson, USA

$12{ }^{3}$ Department of Pharmacology, University of California, San Diego, CA, USA.

$13{ }^{4}$ Skaggs School of Pharmacy and Pharmaceutical Sciences, University of California, San

14 Diego

$15{ }^{5}$ Sydney School of Veterinary Science, University of Sydney, NSW, Australia

16

17 Primary contact: Dr. Richard L. Gallo, e-mail: rgallo@health.ucsd.edu; Tel: 858- 822-4608 


\section{ABSTRACT}

29 Methicillin-resistant Staphylococcus pseudintermedius (MRSP) is an important emerging 30 zoonotic pathogen that causes severe skin infections. To combat infections from drug-resistant 31 bacteria, the transplantation of commensal antimicrobial bacteria as a therapeutic has shown 32 clinical promise. We screened a collection of diverse staphylococcus species from domestic 33 dogs and cats for antimicrobial activity against MRSP. A unique strain ( . felis $\mathrm{C} 4)$ was isolated 34 from feline skin that inhibited MRSP and multiple gram-positive pathogens. Competition experiments in mice showed that $S$. felis significantly reduced MRSP skin colonization and an antimicrobial extract from S. felis significantly reduced necrotic skin injury from MRSP infection. Fluorescence and electron microscopy revealed that $S$. felis antimicrobials disrupted bacterial but not eukaryotic cell membranes. LC/MS identified several $S$. felis phenol-soluble modulin beta (PSM $\beta$ ) peptides that exhibited antimicrobial and anti-inflammatory activity. These findings indicate a feline commensal bacterium that could be utilized in bacteriotherapy against difficult-to-treat animal and human skin infections. 


\section{INTRODUCTION}

Skin is colonized by hundreds of diverse bacterial species that exist within a complex and dynamic chemical landscape. These chemical interactions can play important roles in skin health, immune education and protection against pathogen colonization and infection (Sanford and Gallo, 2013). The composition of the skin microbial community of humans and animals varies extensively, in part due to different skin habitats, i.e. increased hair density in animals, as well as more subtle differences in the chemical and biological conditions of the skin (Grice and Segre, 2011; Ross et al., 2018). Overall, the human microbial skin community is distinct from and significantly less diverse than that of both wild and domestic animals (Ross et al., 2018). Human skin is generally dominated by few taxa present at high abundance e.g. cutibacteria, streptococci and staphylococci, whereas canine skin harbors a more equally distributed and diverse group of taxa (Song et al., 2013). Naturally, close contact between humans and animals can be a source for microbial transmission (Frana et al., 2013; Lai et al., 2017). Although it remains to be determined if shared taxa are stable over time, there are reports that exposure to pets early in life can be protective against atopic disease in later life (Mandhane et al., 2009).

In contrast, there are also many documented cases of human staphylococcal infection from epidemiological exposure to dogs (Somayaji et al., 2016). Companion animals can act as reservoirs for methicillin-resistant $S$. aureus (MRSA) and more commonly, $S$. pseudintermedius (MRSP), with both species sharing many common invasion and virulence factors (Garbacz et al., 2013). The zoonotic significance of S. pseudintermedius may have been previously underestimated because it was frequently misidentified as $S$. aureus in human wound infections (Börjesson et al., 2015). More advanced diagnostic techniques such as MALDI-TOF have led to increased detection of human $S$. pseudintermedius infections (Ference et al., 2019). Colonization of S. pseudintermedius is a contributing factor in canine atopic dermatitis (AD). Interestingly, the prevalence of $\mathrm{AD}$ in humans and $\mathrm{AD}$ in dogs are similar (10-15\% in US) and present with remarkably similar immunological and clinical manifestations (Marsella and Girolomoni, 2009; Silverberg, 2019). Likewise, several studies have reported a decrease in the microbiome diversity of AD and increased colonization of $S$ aureus in humans and S. pseudintermedius in dogs (Fazakerley et al., 2009; Nakatsuji and Gallo, 2019; Older et al., 2020). In human AD, S. aureus was identified in higher relative abundances during disease flares (Kong et al., 2012). Similarly, the relative abundance of $S$. pseudintermedius was also shown to increase with disease flares in canine AD (Bradley et al., 
2016). Common treatment modalities exist for both diseases. Dilute bleach baths are a common antiseptic treatment for $\mathrm{AD}$, with the goal of reducing the carriage of staphylococci (Banovic et al., 2018; Chopra et al., 2017). However, its effectiveness as an antibacterial agent is controversial (Sawada et al., 2019).

An alternative and promising approach is not to disrupt but to re-establish a healthy microbiome community on the skin. To do this our group and others have screened for naturally occurring, and sometimes rare commensal species on healthy human skin that express antimicrobial activity against skin pathogens. A recent example is the discovery of commensal staphylococcus strains that produce lantibiotics that when applied to skin of patients with $\mathrm{AD}$, demonstrated clinically improved symptoms and reductions of lesional $S$. aureus counts (Nakatsuji et al., 2017; Nakatsuji et al. in press). Other studies have reported antimicrobial staphylococcus strains belonging to species of S. lugdunensis (Zipperer et al., 2016), S. epidermidis (Cogen et al., 2010) and S. capitis (O’Neill et al., 2020) isolated from different niches of the skin and nares. In contrast, very little is known regarding the antimicrobial activity of animal-derived staphylococci and their clinical potential against skin infection. Here we identified $S$. felis $\mathrm{C} 4$, a potent antimicrobial isolate from feline skin that inhibited the growth of MRSP in vitro and in vivo. S. felis $\mathrm{C} 4$ produced several $\alpha$-helical amphipathic peptides that demonstrated antimicrobial and anti-inflammatory activity. This discovery represents a potential new bacteriotherapeutic for human and canine skin diseases associated with $S$. pseudintermedius colonization and infection.

\section{RESULTS}

\section{A screen of animal-derived staphylococci isolates identifies a feline skin commensal} bacterium with broad-spectrum antimicrobial activity.

We sought to determine whether commensal staphylococci collected from the skin, nasal, oral and perineal sites of companion dogs and cats exhibit antimicrobial activity against methicillinresistant S. pseudintermedius (MRSP) ST71 (Fig.1A) (Ma et al., 2020; K. Worthing et al., 2018). Fifty-eight staphylococcus isolates across the coagulase-positive (CoPS) and coagulasenegative (CoNS) groups were screened, including validated antimicrobial strains of human origin, S. hominis A9 (Nakatsuji et al., 2017) and S. capitis E12 (O’Neill et al., 2020) and a non-active $S$. aureus 113 negative control strain (Fig.1B). The animal test isolates were screened for antimicrobial activity by live co-culture on agar plates or in the presence of sterile conditioned supernatant, as illustrated in Fig.1A. Amongst all test isolates, five strains 
130 demonstrated greater than $80 \%$ inhibition of $S$. pseudintermedius growth (dashed line) across

131 all three different dilutions of supernatant (1:1, 1:4, 1:8) (Fig.1C). Surprisingly, these strains

132 exhibited greater potency compared to the positive control S. hominis A9 supernatant

133 (indicated by black circle), which inhibited growth of $S$. pseudintermedius in a 1:1 dilution, but

134 not at 1:4 or lower. Amongst the five positive hits, three were identified as $S$. felis and two $S$.

135 pseudintermedius. In the second independent antimicrobial assay, all five isolates including

136 positive control $S$. hominis A9, produced an observable zone of inhibition against $S$.

137 pseudintermedius during live co-culture on agar (Fig.1D). The two feline S. felis species (C4,

138 N26 labelled with white arrows) produced the largest inhibitory zones, extending 3.0-3.3 mm

139 outward from the edge of the growing colony. The S. felis $\mathrm{C} 4$ strain was chosen for further

140 analysis, as it demonstrated potent activity and was isolated from healthy skin. To investigate

141 the significance and selectivity of the S. felis antimicrobial supernatant, we tested its capacity

142 to inhibit the growth of other clinically relevant, gram-positive and gram-negative pathogens

143 (of which several belong to the clinically-relevant ESKAPE group). Of the four gram-negative

144 strains tested, only moderate inhibition was demonstrated after $18 \mathrm{~h}$ incubation with 80-100\%

145 undiluted S. felis C4 supernatant (Fig.1E). In contrast, culture with just 1-5\% of S. felis C4

146 supernatant was sufficient to inhibit $>80 \%$ growth of all four gram-positive organisms,

147 including S. pseudintermedius, E. faecium, B. subtilis and S. aureus.

149 Of the three antimicrobial $S$. felis isolates, only the C4 supernatant retained activity after 150 precipitation with 75\% ammonium sulfate (AS) (Suppl. Fig.1A). Moreover, 75\% AS was 151 highly effective in precipitating the antimicrobial factor(s) from the $\mathrm{C} 4$ supernatant since no 152 activity could be visualized in the non-precipitate fraction (Suppl. Fig.1B). This effect was also 153 achieved with a simpler extraction by n-butanol. The antimicrobial butanol extract remained 154 active at room temperature (RT), up to one week and was stable after boiling (Suppl. Fig.1C). 155 As expected, the butanol extraction provided a partially purified and enriched antimicrobial 156 fraction compared to crude supernatant and AS precipitation (Suppl. Fig1D). Therefore, further 157 experiments involving the $S$. felis $\mathrm{C} 4$ extract describes sterile supernatant obtained via n158 butanol extraction. Interestingly, the antimicrobial activity of $S$. felis $\mathrm{C} 4$ was sufficient to 159 disrupt bacterial biofilms. Reports have shown that most clinically-derived $S$. 160 pseudintermedius strains are biofilm producers (Singh et al., 2013). Biofilm formation is 161 considered an important determinant of staphylococci virulence and is associated with 
A

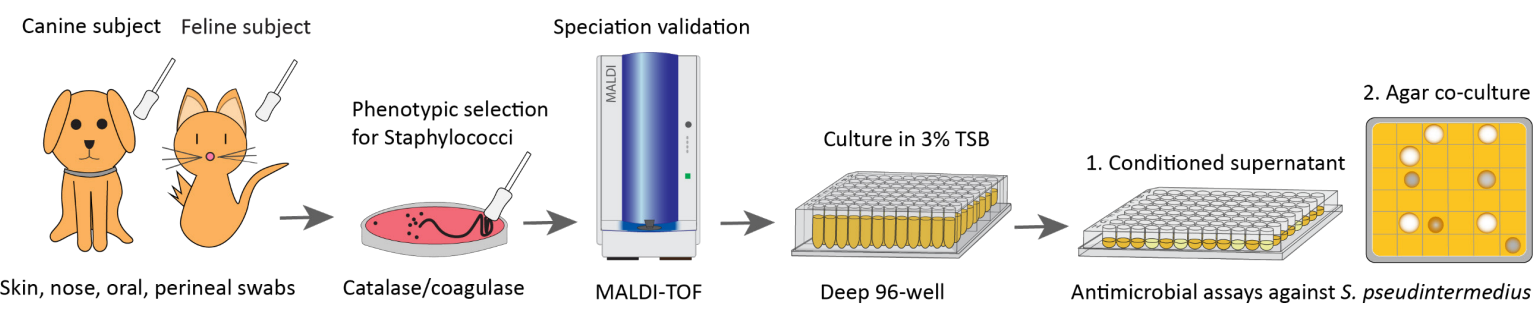

B

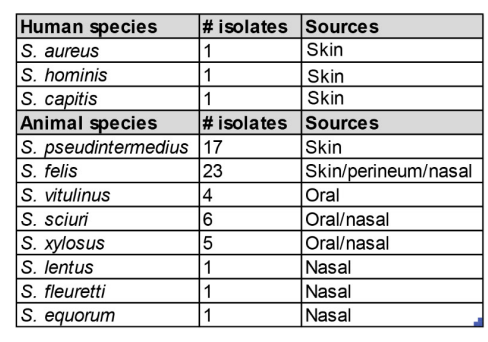

$\mathrm{D}$

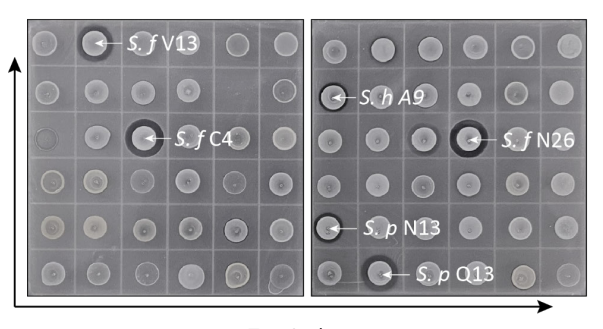

Test isolates
$\mathrm{C}$

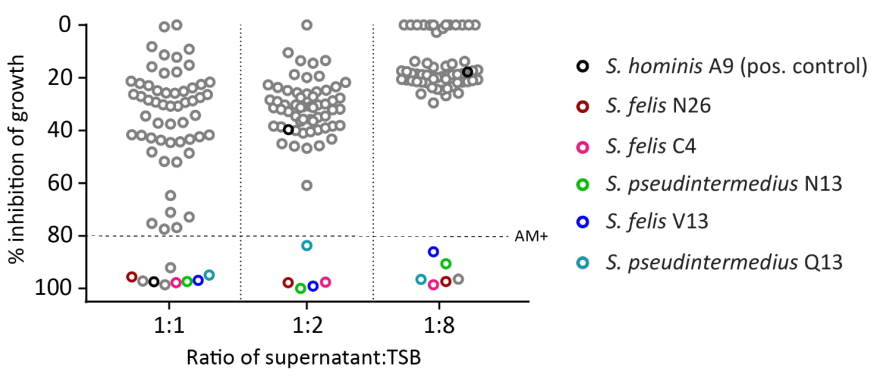

E

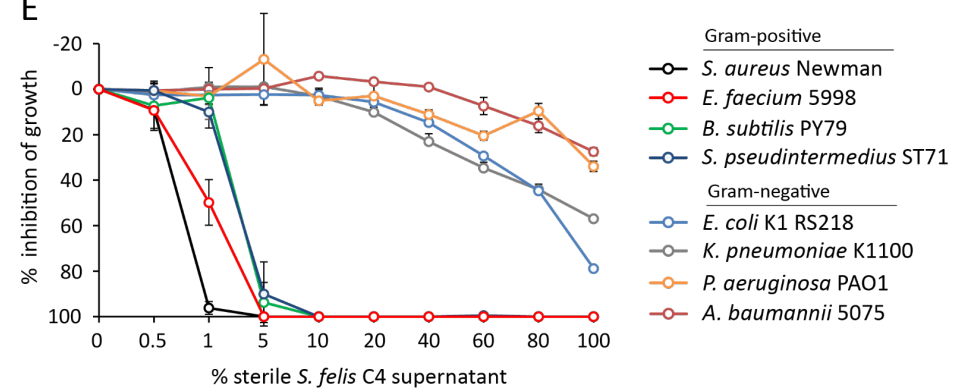

163 Figure 1. Screening and discovery of a feline skin commensal bacterium that inhibits drugresistant gram-positive pathogens.

165 (A) Illustration of the selection and screening strategy of animal-derived staphylococci against the growth of methicillin-resistant S. pseudintermedius (MRSP) ST71 in liquid culture and agar co-culture assays. (B) The panel of 58 feline and canine isolates selected for antimicrobial testing, including human-derived S. hominis A9 and S. capitis E12 positive control antimicrobial strains and the nonantimicrobial S. aureus 113 negative control. (C) Inhibition of S. pseudintermedius ST71 growth by OD600, relative to TSB control at $100 \%$, after $18 \mathrm{~h}$ incubation in $50 \%, 25 \%$, or $12.5 \%(1: 1,1: 4,1: 8$ ratio) sterile conditioned supernatant from all staphylococci isolates. Greater than $80 \%$ inhibition of growth was considered antimicrobial (AM+). (D) Images of the agar co-culture assay showing zone of

173 inhibition (black circle surrounding colony) produced by all staphylococci test isolates against $S$. pseudintermedius ST71, including $S$. felis $\mathrm{C} 4, \mathrm{~N} 26, \mathrm{~V} 13$ (S. $f \mathrm{C} 4, S . f$ N26, $S . f$ V13), $S$. pseudintermedius $\mathrm{N} 13$ and Q13 (S. $p \mathrm{~N} 13$ and $S . p$ Q13), and positive control $S$. hominis A9 (S. $h$ A9, all indicated by arrows). (E) Inhibition of bacterial growth relative to TSB alone at 100\%, against select gram-positive and gram-negative pathogens after $18 \mathrm{~h}$ incubation ( $48 \mathrm{~h}$ incubation for E. faecium) in the presence of increasing amounts of sterile conditioned supernatant from S. felis $\mathrm{C} 4$ overnight growth. Error bars indicate SEM. Representative of three separate experiments. 
increased skin colonization and severity of disease (Di Domenico et al., 2018; Kwiecinski et al., 2015). A 4 h preformed biofilm of pseudintermedius ST71 showed a significant decrease in crystal violet $(\mathrm{CV})$ staining over time, when incubated with $100 \%$ conditioned supernatant of $S$. felis $\mathrm{C} 4$, indicating biofilm disruption and degradation (Suppl. Fig. 2A). Importantly, the S. felis $\mathrm{C} 4$ extract also exhibited similar anti-biofilm activity as crude conditioned supernatant, with biofilm mass reduced by $48 \%$ at $250 \mu \mathrm{g} / \mathrm{ml}$ and $58 \%$ at $500 \mu \mathrm{g} / \mathrm{ml}$ (Suppl. Fig.2B).

\section{S. felis $\mathbf{C} 4$ inhibits $S$. pseudintermedius skin colonization and infection in mice.}

We next sought to investigate the translational potential of S. felis $\mathrm{C} 4$ and its antimicrobial products as a potential therapy against $S$. pseudintermedius colonization and infection. Since S. felis $\mathrm{C} 4$ is a commensal bacterium that was isolated from healthy feline skin, we speculated it should be safe and well tolerated on mouse skin. S. felis $\mathrm{C} 4$ was found to be sensitive to several common antibiotics (Fig. 2A) and as such represents a suitable strain for further investigation as a bacteriotherapy. We therefore assessed the skin tolerability of a 3-day topical application of S. felis $\mathrm{C} 4$ on SKH1 hairless mice. Whereas $S$. pseudintermedius and the nonantimicrobial S. felis ATCC 49168 induced evidence of scaling and redness on mouse back skin, S. felis C4 did not promote any adverse reaction (Fig.2B).

To test the antimicrobial activity of $S$. felis $\mathrm{C} 4$ on skin, we applied $5 \times 10^{7} \mathrm{CFU} / \mathrm{cm}^{2} S$. pseudintermedius directly onto mouse back skin for $48 \mathrm{~h}$, then applied an equal density of $S$. felis $\mathrm{C} 4$ or $1 \mathrm{mg}$ of extract to the infected site. This was repeated daily for three days. The back skin showed a reduction in scaling and redness post-treatment with $\mathrm{S}$. felis $\mathrm{C} 4$ or extract compared to control (Fig. 2C). Enumeration of S. pseudintermedius ST71 CFUs after plating onto selective agar revealed a significant $2.9 \mathrm{log}$ decrease in CFU after extract application and a $3.3 \log$ decrease after live S. felis C4 application (Fig. 2D). Enumeration of total staphylococci CFUs after plating onto selective agar once again confirmed that the extract treatment significantly reduced bacterial colonization (Fig.2E). In contrast, total staphylococci CFU counts after the $S$. felis $\mathrm{C} 4$ application were more similar to the control group, suggesting that the $S$. felis bacteria successfully colonized and outcompeted S. pseudintermedius ST71 on the skin (Fig. 2E). To further test S. felis $\mathrm{C} 4$ extract as an anti-MRSP intervention, we evaluated its efficacy in limiting the infectious outcome of cutaneous challenge with S. pseudintermedius. An inoculum of $1 \times 10^{7}$ CFU S. pseudintermedius ST71 was intradermally administered into the back skin of mice. At $1 \mathrm{~h}$ post-infection, two intradermal inoculations of $250 \mu \mathrm{g}$ extract were administered adjacent to the infection site and necrosis was monitored by measuring 


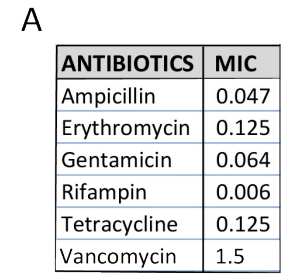

C

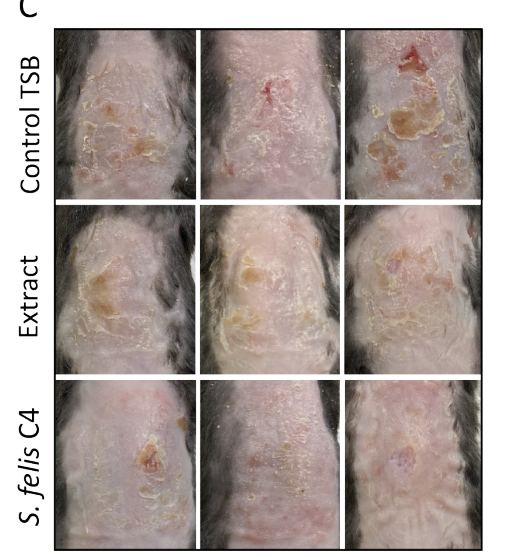

$\mathrm{F}$

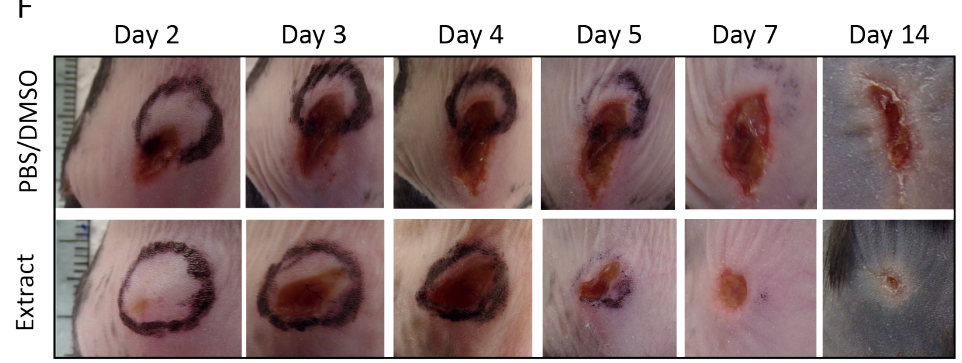

B

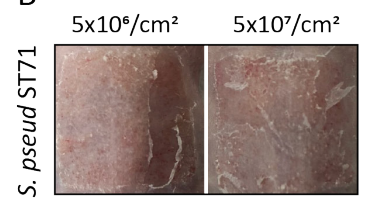

D

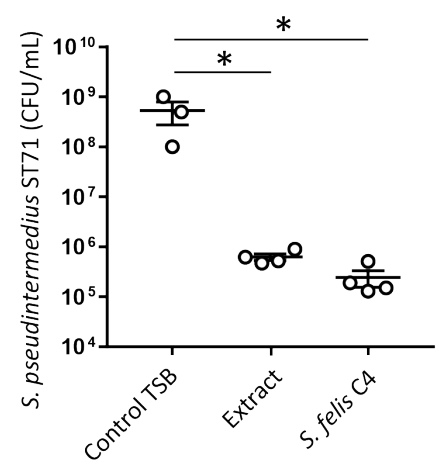

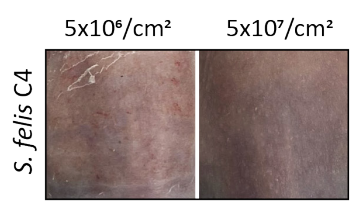

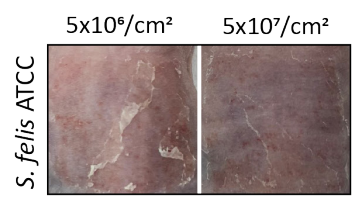

G

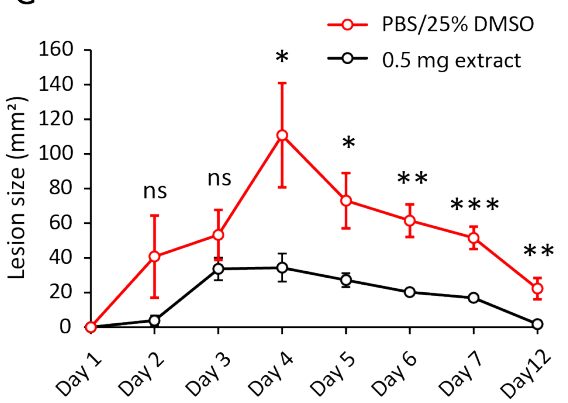

215 Figure 2. Live bacteriotherapeutic intervention with $S$. felis C4 protects against $S$. 216 pseudintermedius colonization in mice.

217 (A) Minimum inhibitory concentrations (MIC) of the indicated antibiotics against S. felis C4. (B)

218 Representative images of the dorsal skin of 8-10 week-old SKH1 mice 3 days post-challenge with live

219 S. felis C4, S. pseudintermedius ST71 (S. pseud ST71) or S. felis ATCC 49168, inoculated at the 220 indicated amounts. $\mathrm{n}=2$, per treatment group (C-E) $5 \times 10^{7} \mathrm{CFU} / \mathrm{cm}^{2}$ of $S$. pseudintermedius ST71 was applied onto the back skin of C57BL/6 mice for $48 \mathrm{~h}$ and challenged with TSB, S. felis C4 extract (1 $\mathrm{mg}$ ) or live $\mathrm{S}$. felis $\mathrm{C} 4\left(5 \times 10^{7} \mathrm{CFU} / \mathrm{cm}^{2}\right)$ for $72 \mathrm{~h}$. Post-treatment, mouse back skin was photographed (C) and swabbed to enumerate S. pseudintermedius ST71 CFU on selective Baird-Parker egg yolk tellurite agar (D) or total staphylococci CFU on selective mannitol-salt agar plates (E). $n=3$ for TSB and $\mathrm{n}=4$ for extract and $S$. felis $\mathrm{C} 4$. Error bars indicate SEM. One-way ANOVA with multiple comparisons (Dunnett's correction) was performed. $p$ values: ${ }^{*} p<0.05$; (F-G) At day $0,1 \times 10^{7} \mathrm{CFU}$ of $S$. pseudintermedius ST71 was intradermally injected into the back skin of 8-10 week-old C57BL/6 mice and at $1 \mathrm{~h}$ post-infection two inoculations of $S$. felis C4 extract $(250 \mu \mathrm{g})$ or PBS/25\% DMSO control were injected adjacent to the infection site. (F) Representative images of $S$. pseudintermedius 
231 Quantification of lesion size $\left(\mathrm{mm}^{2}\right)$ over time as measured by $\mathrm{L} \times \mathrm{W}$ of lesions. $\mathrm{n}=4$ for DMSO/PBS

232 and $\mathrm{n}=5$ for extract. Error bars indicate SEM. A two-tailed, unpaired Student's $t$-test was performed. $p$

233 values: $* p<0.05 ; * * p<0.01 ; * * * p<0.001$.

lesion size over a 14-day period. Compared to controls, the extract-treated mice exhibited slower lesion progression from day 1 to 2 , and significantly better protection from $S$. pseudintermedius skin disease from day 4 (Fig.2F-G). These results demonstrate the in vivo efficacy and clinical potential for $S$. felis $\mathrm{C} 4$ as a bacteriotherapy against $S$. pseudintermedius skin colonization and infection.

\section{Antimicrobial S. felis $\mathbf{C} 4$ promotes disruption of the bacterial membrane.}

Next, we sought to better understand how the antimicrobial action of $S$. felis C4 negatively affects bacterial physiology. Given the selective nature of the S. felis $\mathrm{C} 4$ supernatant against gram-positives (Fig.1E), we speculated that S. felis $\mathrm{C} 4$ activity may target and compromise the bacterial membrane and/or cell wall. To address this question, transmission electron microscopy (TEM) imaging was conducted on sectioned S. pseudintermedius ST71 bacteria exposed to $1 \mathrm{~h}$ treatment with control DMSO (1\%), sub-MIC $(1 \mu \mathrm{g} / \mathrm{ml})$, MIC $(8 \mu \mathrm{g} / \mathrm{ml})$ or $5 \mathrm{X}$ MIC (40 $\mu \mathrm{g} / \mathrm{ml})$ extract concentrations (Fig.3A). TEM observations upon control DMSO treatment showed normal, uniform spherical cocci physiology and septum formation indicating active replication. In contrast, short exposure to the extract resulted in drastic changes to the bacterial ultrastructural morphology, with evidence of cell wall thickening and alterations in the structure and rigidity of the cell membrane (Fig.3A, lower zoom inset panels). Moreover, treatment with extract showed evidence of greater chromosomal compaction compared to control, evidenced by the increased (electron) density of the nucleoid (highlighted yellow arrows). Additional evidence supporting a role for S. felis $\mathrm{C} 4$ in promoting bacterial membrane damage was provided by several in vitro microbial cell viability assays. From mid-log phase cultures of $S$. pseudintermedius ST71 30 min post-treatment with the extract, a dose-dependent increase in reactive oxygen species (ROS) (Fig.3B) coincided with a concomitant decrease in intracellular ATP levels (Fig.3C). Further evidence for a membrane-active antimicrobial was provided by increased dual staining of SYTO9-positive bacteria (green) with the membraneimpermeable dye propidium iodide (PI) (red). After treatment with 5X MIC of extract, a proportion of bacterial cells showed dual staining, with entry of PI likely reflecting a loss of membrane integrity (Fig. 3D). 

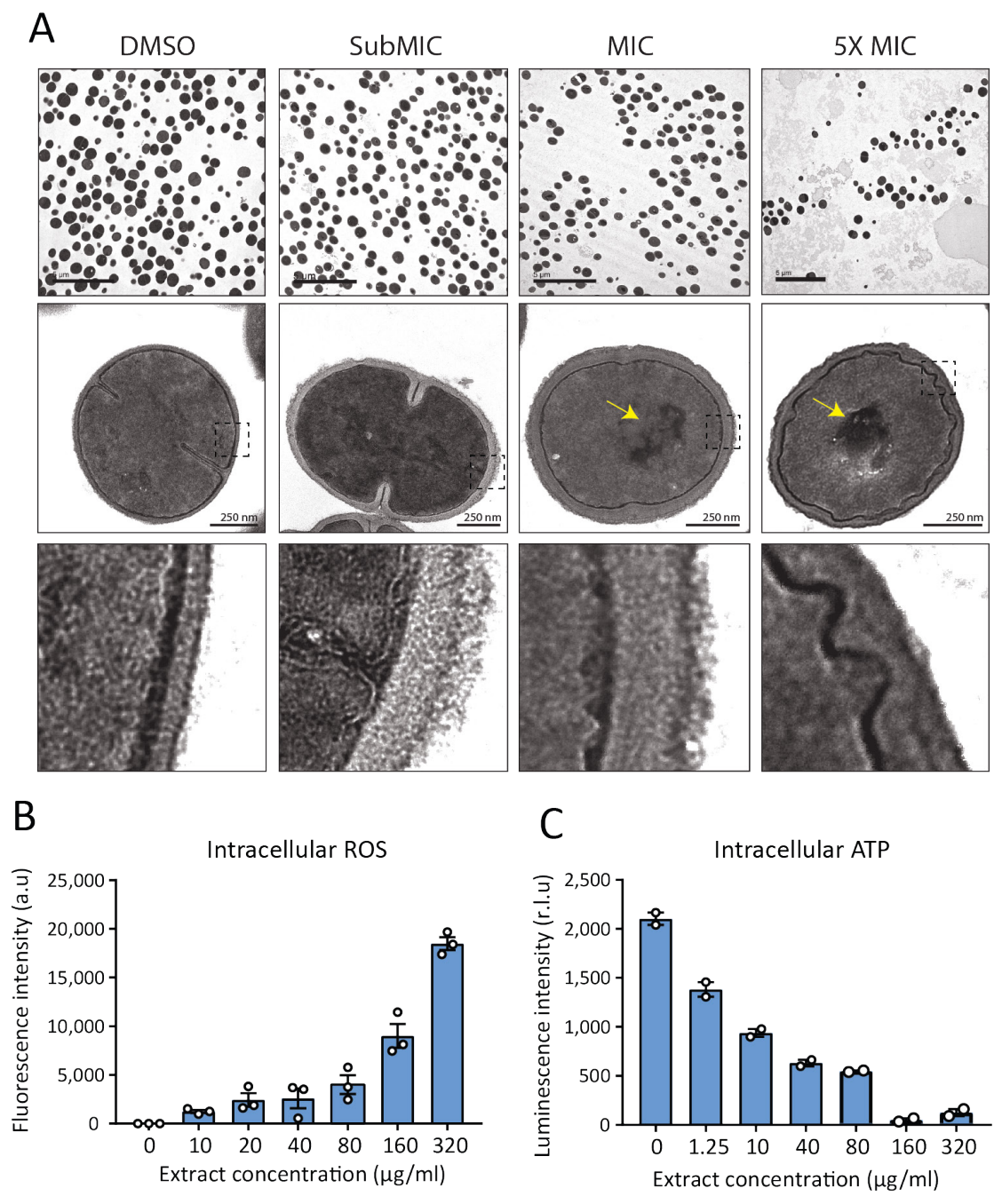

\section{D}

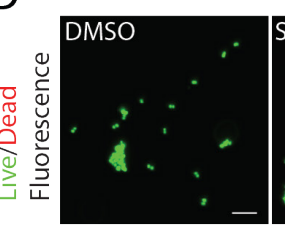

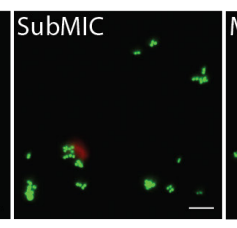
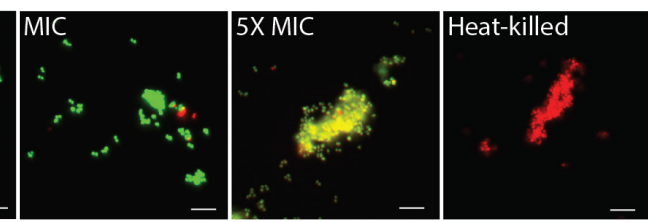

Figure 3. S. felis $\mathrm{C} 4$ extract contains a membrane-active antibacterial molecule.

268 (A) TEM images of $S$. pseudintermedius ST71 after $1 \mathrm{~h}$ treatment with DMSO control or S. felis C4 extract at indicated concentrations. Yellow arrows indicate evidence of condensed DNA. Lower image panels represent higher magnification of regions highlighted by dashed black boxes. Scale $=250 \mathrm{~nm}$ (B) Total ROS accumulation in S. pseudintermedius ST71 after $1 \mathrm{~h}$ treatment in increasing concentrations of S. felis $\mathrm{C} 4$ extract. Error bars indicate SEM. (C) Total intracellular ATP accumulation 273 in S. pseudintermedius ST71 after $1 \mathrm{~h}$ treatment in increasing concentrations of S. felis $\mathrm{C} 4$ extract. Error 274 bars indicate SEM. (D) Live/dead fluorescent images of S. pseudintermedius ST71 after $1 \mathrm{~h}$ treatment 275 with DMSO control or extract at indicated concentrations. Viable bacterial cells were stained green by 276 SYTO9 and damaged/dead cells were stained red by PI. Scale $=10 \mu \mathrm{m}$. (B), (C) representative of two 277 separate experiments. 
Purification and identification of PSM $\beta$ peptides as antimicrobial products of $S$. felis $\mathrm{C} 4$.

To determine the nature of the antimicrobial product produced by $S$. felis $\mathrm{C} 4$, sterile supernatant was purified by HPLC. This yielded two major peaks that eluted at $44 \%$ and $47 \%$ acetonitrile (Suppl. Fig.3A). Anti-S. pseudintermedius activity was predominantly associated with fraction 32 which eluted at 55\% acetonitrile (Suppl. Fig.3B). SDS PAGE and silver stain of fraction 32 and neighboring inactive fractions revealed a unique band of roughly $5 \mathrm{kDa}$ in size (Suppl. Fig.3C). To determine if this small protein was responsible for antimicrobial activity, gel slices of the fraction 32 lane corresponding to small, medium and larger proteins $(\leq 5 \mathrm{kDa}, 5-20 \mathrm{kDa}$ and 20-50 kDa, respectively) were excised and extracted by acetone precipitation, as previously described (Botelho et al., 2010; Zhang et al., 2015). Only the $\leq 5 \mathrm{kDa}$ band demonstrated antimicrobial activity after incubation with S. pseudintermedius (Suppl. Fig.3D), thereby suggesting the likely candidate to be a small peptide. Mass spectrometry (MS) analysis of the top 8 hits in the active and non-active fractions identified several putative small antimicrobial peptides (AMP), representing the phenol soluble modulin beta (PSM $\beta 1-3)$ and gamma (PSM $\gamma$, aka delta-hemolysin) families and a peptide of unknown function containing a EF-hand domain, common amongst some antimicrobial $\mathrm{Ca}^{2+}$ binding proteins, such as $\mathrm{S} 100 \mathrm{~A} 8 / \mathrm{S} 100 \mathrm{~A} 9$ (Chazin, 2011) (Suppl. Fig.3E). Whole genome analysis of the S. felis C4 strain confirmed the presence of three PSM $\beta$-encoding genes (Suppl. Fig.3F). Like some mammalian cationic AMPs such as cathelicidin LL-37, the PSM $\beta$ and EF-hand domain peptides have an $\alpha$-helical amphipathic-like formation, a structural motif conserved in the recognition and binding of biological membranes (Suppl. Fig.3G). To determine if the peptides identified by MS exhibited antimicrobial activity, synthetic versions of all three $S$. felis PSM $\beta 1-3$ and the EF-hand domain containing peptide were tested. Interestingly, all three PSM $\beta$ peptides inhibited $S$. pseudintermedius growth at a concentration of $50 \mu \mathrm{g} / \mathrm{ml}$ (Fig.4A). In contrast, the EF-hand domain-containing peptide did not inhibit bacterial growth up to $200 \mu \mathrm{g} / \mathrm{ml}$. These results suggest that PSM $\beta$ peptides could be mediating the antimicrobial activity of $S$. felis $\mathrm{C} 4$.

\section{S. felis C4 extract and PSM $\beta$ peptides exhibit anti-inflammatory activity by suppressing} TLR-mediated inflammation.

307 Unlike the well characterized cytolytic and inflammatory activities of PSM $\alpha$, a defined role for 308 PSM $\beta$ in mediating host interactions has been largely unexplored (Da et al., 2017). Based on our previous observation that $\mathrm{S}$. felis $\mathrm{C} 4$ was well tolerated on murine skin, we asked if the 
311 Primary normal human keratinocytes (NHEK) were treated with increasing concentrations of

312 different PSMs for $24 \mathrm{~h}$ and cytotoxicity determined by quantifying LDH release. As expected,

313 the hemolytic toxins PSM $\alpha$ and PSM $\gamma$ were found to be highly cytotoxic whereas S. felis

314 PSM $\beta 2$, PSM 33 , and the extract resulted in less than 5\% LDH release at the antimicrobial

315 concentration of $50 \mu \mathrm{g} / \mathrm{ml}$ (Fig.4B).

316

A

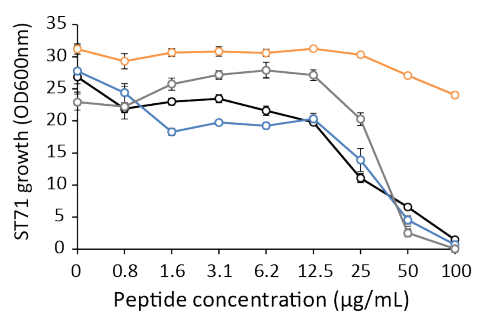

C

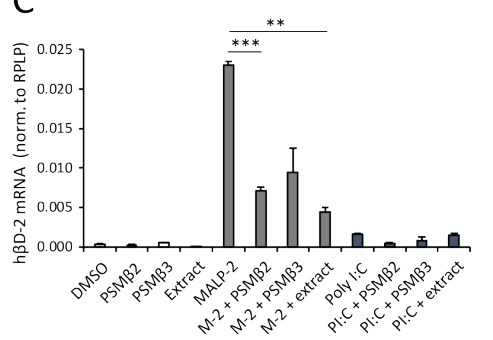

D

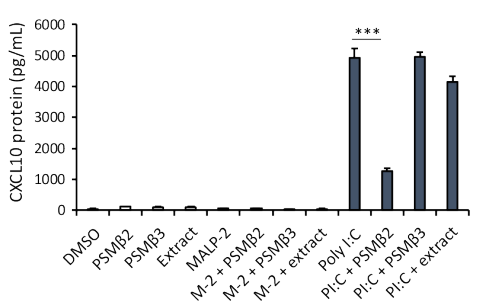

G
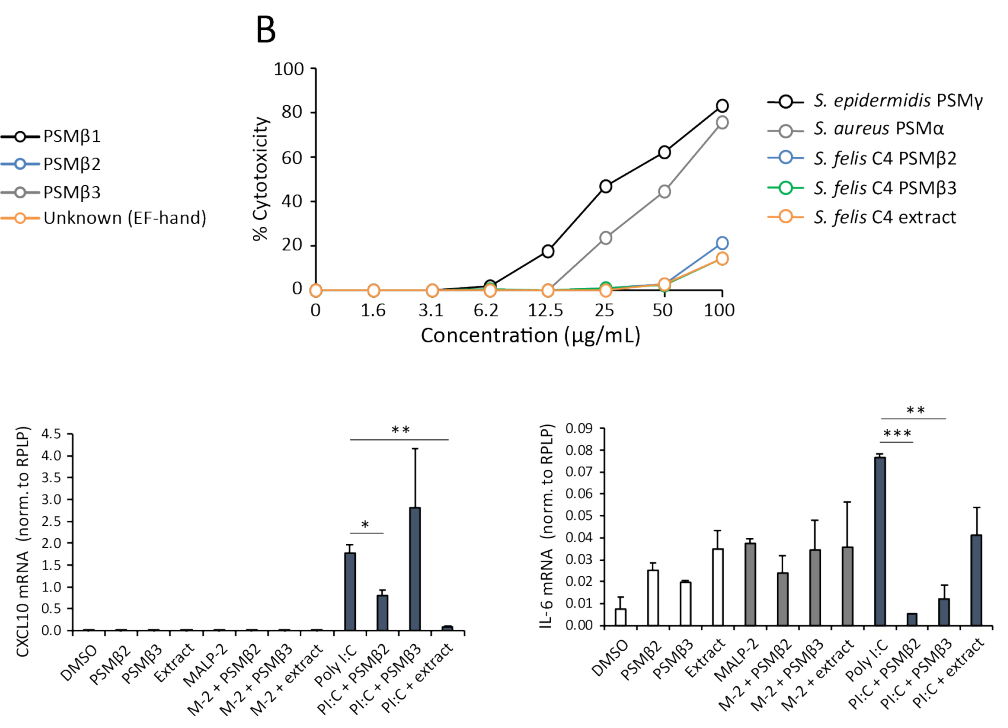

E

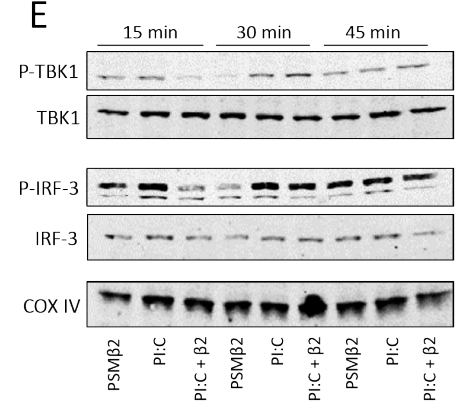

F

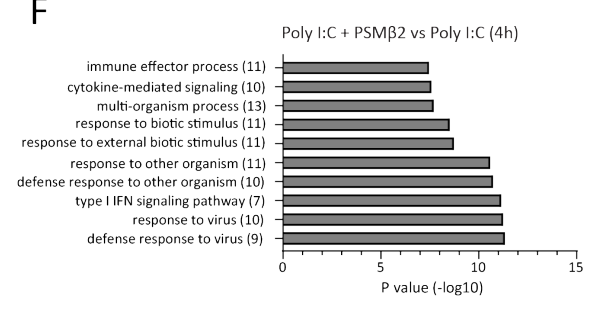

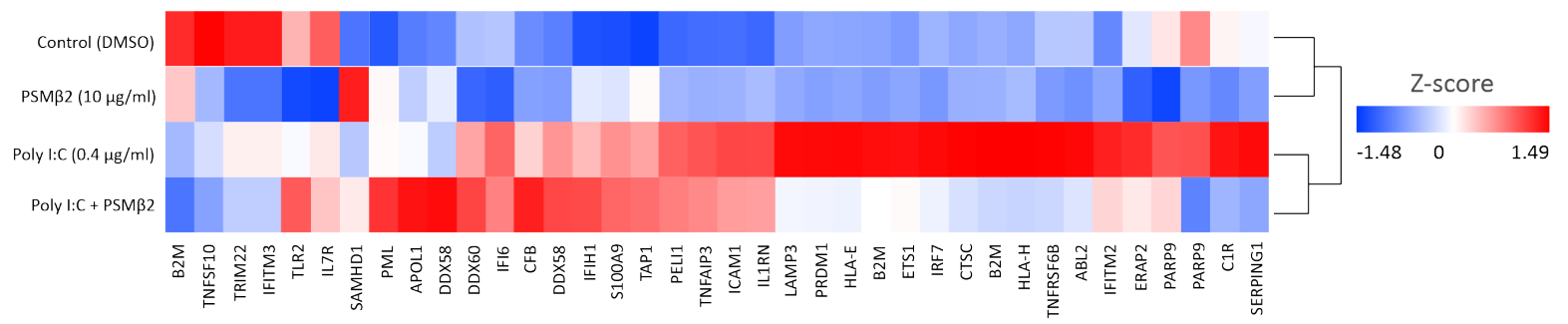

Figure 4. Antimicrobial S. felis C4 extract and PSM $\beta$ suppress TLR-mediated inflammation.

320 (A) Growth of S. pseudintermedius ST71 after $18 \mathrm{~h}$ incubation with increasing concentrations of 321 synthetic formylated PSM $\beta$ peptides (PSM $\beta 1$, PSM $\beta 2$, PSM $\beta 3$ ), or synthetic peptide containing an EFhand domain (Unknown EF-hand). (B) Lactate dehydrogenase (LDH) release in NHEKs after $24 \mathrm{~h}$ treatment with $S$. felis $\mathrm{C} 4$ extract, $S$. felis PSMß2, PSMß3 or positive control cytotoxic PSM $\alpha$ from $S$. 
aureus or PSM $\gamma$ from S. epidermidis. Percentage (\%) cytotoxicity measured by maximum LDH release into supernatant collected after untreated cell freeze thaw. (C) mRNA transcript abundance measured by qPCR in NHEKs stimulated with or without TLR2/6 agonist MALP-2 $(200 \mathrm{ng} / \mathrm{ml})$ or TLR3 agonist Poly I:C $(0.4 \mu \mathrm{g} / \mathrm{ml})$ in the presence or absence of $S$. felis C4 extract, PSM $\beta 2$ or PSM $\beta 3(10 \mu \mathrm{g} / \mathrm{ml})$ or DMSO control $(0.1 \%)$ at $4 \mathrm{~h}$ post-treatment. (D) Quantification of CXCL10 protein by ELISA from supernatant of NHEKs stimulated with MALP-2 or Poly I:C in the presence of S. felis extract or PSM $\beta 2$, PSM 33 or DMSO control $24 \mathrm{~h}$ post-treatment. (E) Time-course of the TLR3 signaling cascade by immunoblot of phosphorylated TBK1 and IRF3 proteins after stimulation of NHEKs with Poly I:C, DMSO, or co-treatment with Poly I:C and PSMß2. (F) Gene ontology (GO) pathway analysis of genes downregulated in NHEKs after $4 \mathrm{~h}$ co-treatment with Poly I:C and PSM $\beta 2$ versus treatment with Poly I:C alone. (G) Hierarchical clustering and Heatmap visualization of selected genes from GO enriched 'immune response' pathway (1.5-fold change) $4 \mathrm{~h}$ post-treatment with DMSO, PSM $\beta 2$ or Poly I:C alone or with PolyI:C and PSM 22 cotreatment. (A-D) error bars indicate SEM. Representative of at least three separate experiments. One-way ANOVA with multiple corrections (Turkey correction) was performed. $p$ values: ${ }^{*} \mathrm{p}<0.05 ; * * \mathrm{p}<0.01 ; * * * \mathrm{p}<0.001$.

To investigate the potential effects of $\mathrm{S}$. felis $\mathrm{C} 4$ on the host immune response, we stimulated cells with various TLR agonists in the presence or absence of the extract or the individual PSMs and measured inflammatory gene expression. NHEKs were treated with S. felis PSM $\beta 2$, PSM $\beta 3$, extract, DMSO control alone, or each in combination with the TLR2 agonist MALP$2(200 \mathrm{ng} / \mu \mathrm{l})$ or the TLR3 agonist Poly I:C $(0.4 \mu \mathrm{g} / \mathrm{ml})$ for $4 \mathrm{~h}$. Neither PSM $\beta$ nor the extract resulted in any detectable increase in gene expression, whereas MALP-2 significantly increased the expression of hBD-2, and Poly I:C significantly increased CXCL10 and IL-6 expression (Fig.4C). Interestingly, these TLR-mediated responses were significantly reduced during co-treatment with PSM $\beta$ or extract. This result was confirmed by ELISA, showing that PSM $\beta 2$ had a significant effect on suppressing CXCL10 secretion in NHEKs after $24 \mathrm{~h}$ cotreatment (Fig.4D). To determine if this interaction is specific for epithelial cells, we also stimulated human THP-1 macrophage-like cells with MALP-2, or the TLR4 agonist LPS and found that IL-6 and TNF $\alpha$ expression was decreased during co-treatment with PSM $\beta 2$ (Suppl.

353 Fig.4). The addition of PSM $\beta 2$ to NHEK activated by poly I:C demonstrated that PSM $\beta 2$ 354 inhibited phosphorylation of TBK1 and IRF3 at 15 min post-stimulation with the peptide (Fig.4E). This inhibition of inflammatory target gene and kinase activity was further evaluated

356 by the analysis of changes in global gene expression using RNA-Seq analysis of NHEKs 357 stimulated with Poly I:C, with and without PSM $\beta 2$ at $4 \mathrm{~h}$ and $24 \mathrm{~h}$. Gene ontology (GO) 
analyses revealed the significant down-regulation of several gene clusters associated with 'immune effector process' and 'type I IFN signaling' at $4 \mathrm{~h}$ during co-treatment of PSM $\beta 2$ and Poly I:C (Fig.4F). A heatmap of selected genes within the 'Immune response' GO term at $4 \mathrm{~h}$ post-treatment further highlighted the suppressive effect, but more significantly, showed that PSM $\beta 2$ treatment alone did not induce an immunological response in NHEKs (Fig.4G). However, when NHEKs were treated with PSM $\beta 2$ in the absence of an inflammatory stimulus, we identified the downregulation of genes within GO terms such as "response to interleukin1" (Suppl. Fig.5A) and "pathogenic E. coli infection" (Suppl. Fig.5B), suggesting that exposure to PSM $\beta 2$ primes cells to dampen potential inflammatory mediators in response to bacterial ligands. This contrasted with PSM $\beta 2$-upregulated genes which were mostly associated with biosynthetic pathways including lipid and amino acid metabolism (data not shown).

\section{DISCUSSION}

S. pseudintermedius is one of the most common pathogens isolated from the skin of dogs and becoming increasingly prevalent on humans. The incidence of severe and recurrent infections in animals and humans caused by methicillin-resistant $S$. pseudintermedius is increasing and is associated with a predominant clone belonging to the multi-locus sequence type ST71 (Darlow et al., 2017; Perreten et al., 2010; Riegel et al., 2011; Robb et al., 2017; Stegmann et al., 2010; Weese et al., 2009). ST71 isolates exhibit resistance to many classes of antibiotics and represent a considerable therapeutic challenge. Our group has made advancements in the discovery and characterization of skin antimicrobial isolates that are effective against drugresistant pathogens and can be applied as a novel therapeutic (Cogen et al., 2010; Nakatsuji et al., 2018, 2017; O'Neill et al., 2020). Here we followed a similar discovery pathway, screening for antimicrobial activity in isolates from domestic cats and dogs that could inhibit the growth of $S$. pseudintermedius. We selected an isolate from feline skin that secreted small peptides which were easily extractable by established biochemical methods for an antimicrobial extract preparation. The application of either treatment modality onto infected mouse skin greatly reduced MRSP infection and colonization. These results highlight the clinical potential for transplantation of antimicrobial commensals to treat skin infections that are recalcitrant to classic antibiotic treatment.

There are several reports of the discovery and characterisation of distinct antimicrobialproducing strains within common human bacterial skin species, including S. epidermidis (Cogen et al., 2010) S. hominis (Nakatsuji et al., 2017), S. lugdunensis (Zipperer et al., 2016), 
392 S. capitis (O’Neill et al., 2020) and recently Cutibacterium acnes (Claesen et al., 2020).

393 Unfortunately, the frequency and abundance of such antimicrobial isolates are found to be 394 significantly reduced during human $\mathrm{AD}$ and S. aureus colonization (Nakatsuji et al., 2017).

395 Indeed, this phenomenon could also influence canine AD, which shares many of the clinical 396 features of human $\mathrm{AD}$ and a corresponding predisposition to $S$. pseudintermedius colonization.

397 We hypothesized that the screening of poorly characterized bacterial species from diverse body 398 sites of healthy animals could be a promising strategy to discover new antimicrobial strains 399 that are effective against zoonotic pathogens. Following the high-throughput antimicrobial 400 screening of a collection of diverse animal-derived staphylococci, we discovered S. felis C4, 401 which secretes antimicrobials targeting MRSP and other clinically relevant, drug-resistant 402 gram-positive pathogens. S. felis remains poorly characterized in the literature, but is the most 403 frequent species of staphylococci isolated from cats and is susceptible to most antimicrobials 404 (K. Worthing et al., 2018). In this study, twenty-three S. felis isolates were screened but only 405 three showed reproducible antimicrobial activity in liquid and agar co-culture, suggesting an uncommon intraspecies trait. Importantly, we demonstrated that topical application of the live S. felis C4 organism outcompeted MRSP colonization in vivo, likely by the active secretion of its antimicrobials on the skin surface. Indeed, topical application of the sterile extract was similarly effective in reducing CFU counts on mouse skin. Injection of the extract during $S$. pseudintermedius skin infection was also effective in significantly reducing the size of necrotic lesions. These positive in vivo findings build upon other reports of the utilization of commensal bacteria as biotherapeutic products to treat skin diseases.

414 In the treatment of $\mathrm{AD}$, it has been suggested that the topical application of a $5 \%$ lysate from 415 the gram-negative bacterium Vitreoscilla filiformis can be effective in human and mouse AD 416 models (Gueniche et al., 2008). Another group reported improved outcomes in mice and on 417 humans when another commensal gram-negative bacterium called Roseomonas mucosa was 418 applied to their skin. Although gram-negative bacteria do not typically survive on the skin surface, this strain was isolated from the skin of a healthy human volunteer, suggesting transient colonization from environmental exposure (Myles et al., 2019). Our group has sought to isolate potentially protective bacteria from taxa that have evolved the capacity to survive on the skin, thus maximizing the capacity to attack pathogen targets. We have recently shown that S. aureus colonization is reduced after transplantation of a human commensal S. hominis A9 strain onto AD patient skin in a randomized, double-blind, placebo-controlled trial (Nakatsuji et al., 2017; Nakatsuji et al. in press). This anti-S. aureus activity was mediated by several 
secreted lantibiotics unique to $S$. hominis A9. Typically, lantibiotics and bacteriocins are produced by multimodular enzymes of the nonribosomal peptide synthetases and polyketide synthases (Walsh, 2008). Unfortunately, their complex structure and chemistry are major hurdles to their adoption in the clinic. Furthermore, they are difficult to purify and synthesize, and are unstable and sensitive to proteases, $\mathrm{pH}$, and oxidation (Ross and Vederas, 2011). Alternatively, small $\alpha$-helical AMPs, which include mammalian cathelicidin (active LL-37) and defensins (Nakatsuji and Gallo, 2012) as well as bacterial PSMs (Cogen et al., 2010; Zeng et al., 2019), could be better candidates.

The family of phenol-soluble modulins which include PSM $\alpha$, PSM $\beta, \operatorname{PSM} \gamma$, and PSM $\varepsilon$, are a class of small, immunomodulatory AMPs found in many staphylococci species. PSM $\alpha$ and $\operatorname{PSM} \gamma$ are classic cytolytic toxins but PSM $\beta$ does not exhibit cytotoxic activity against eukaryotic membranes despite a strong affinity to bind and lyse POPC vesicles, which mimic biological membranes (Duong et al., 2012). As a result, the biological function of PSM $\beta$ has remained unclear (Cheung et al., 2014). However, several recent studies have reported the antimicrobial activity of PSM $\beta$ (Kumar et al., 2017; O’Neill et al., 2020). Here, we detected several PSM $\beta$ peptides by MS, that were highly enriched in an antimicrobial HPLC fraction from $S$. felis $\mathrm{C} 4$ supernatant. Their activity against the growth of MRSP was validated using synthetic versions of the peptides, but they exhibited less potency than the extract. As such, due to the partially purified nature of the extract, the contribution of other antimicrobials products cannot be ruled out. This could include the AMP PSM $\alpha$ which is known to co-elute with PSM $\beta$ by HPLC, but is difficult to detect and not well annotated in bacterial genomes due to its small size (Cheung et al., 2014; Joo et al., 2011). Nevertheless, both fluorescence and electron microscopy of bacteria exposed to the extract showed drastic perturbations of the bacterial cell membrane and cell wall thickening, which is consistent with the membranetargeting actions of amphipathic AMPs. The concomitant accumulation of bacterial ROS and decrease in ATP production are also consistent with bacterial membrane disruption and increased permeability (Song et al., 2020). Future research efforts will attempt to develop and characterize truncated and mutated versions of $S$. felis PSMs with the goal of enhancing antimicrobial activity for a more simplistic but potentially more powerful therapeutic (Zeng et al., 2019).

In addition to their protection against pathogen colonization, skin commensals play important roles in promoting skin health and immune homeostasis. Although PSMs are common amongst 
staphylococci, pathogenic S. aureus exhibits a preference for PSM $\alpha$ production over PSM $\beta$. In contrast, commensal staphylococci production of PSM $\beta$ is prioritized over the more toxic PSM $\alpha$ and PSM $\gamma$ versions, a feature suspected to be an evolutionary adaptation to stably colonize skin (Otto, 2009; Wang et al., 2007). Naturally, S. felis C4 PSM $\beta$ and extract treatment of NHEKs yielded minimal evidence of cytotoxicity, whereas PSM $\alpha$ and PSM $\gamma$ induced extensive cytotoxicity. These smaller PSMs are well characterised toxins that trigger proinflammatory responses (Nakamura et al., 2013; Williams et al., 2019). Whereas the larger PSM $\beta$ reportedly does not elicit pro-inflammatory activity in vitro - a finding that was supported by our data, little is known regarding other potential host responses to PSM $\beta$ exposure (Cheung et al., 2014). We speculated whether PSM $\beta$ might exhibit anti-inflammatory activities, and in the present context that activity would be therapeutically beneficial. Indeed, when both NHEKs and THP-1 macrophages were treated with S. felis PSMs or extract in the presence of TLR agonists, cytokine induction was reduced but most evidently by PSM $\beta 2$. RNA-Seq analysis of NHEKs revealed global suppression of inflammatory pathways typically activated by TLR3, in the presence of PSM $\beta 2$. Treatment of NHEKs with PSM $\beta 2$ alone showed the downregulation of genes associated with IL- 1 and bacterial infection, suggesting that PSM $\beta$ promotes tolerance to commensal microbes. Here we report that $S$. felis $\mathrm{C} 4$ bacterium is an attractive biotherapeutic candidate for skin disease, that could benefit patients due to established low cytotoxicity and its broad-spectrum antimicrobial activity and antiinflammatory activity.

\section{MATERIALS AND METHODS}

\section{Bacterial strains and growth conditions}

483 The bacterial strains used in this study were all grown overnight, with the exception of $E$. 484 faecium which was grown for 48 h, in Tryptic Soy Broth (TSB) (Oxoid) with shaking or on agar at $37{ }^{\circ} \mathrm{C}$ under static conditions.

\section{Sample collection}

488 Animal-derived staphylococci samples came from two previously described collections: the first collection consisted of clinical isolates of skin and soft tissue infection from Australian dogs and cats (K. A. Worthing et al., 2018), and the second collection was comprised of staphylococci isolated from the nose, mouth and perineum of healthy dogs and cats in Australia

492 (Ma et al., 2020). All samples had previously been identified by matrix assisted laser desorption 493 ionization-time of flight mass spectrometry (MALDI-TOF), as previously described (K. 
Worthing et al., 2018), and the ST71 MRSP isolate had been characterized by whole genome sequencing (K. A. Worthing et al., 2018). Two human derived antimicrobial skin commensal isolates were used as positive controls: S. hominis A9 (Nakatsuji et al., 2017) and S. capitis E12 (O’Neill et al., 2020) and a non-antimicrobial S. aureus 113 isolate served as a negative control.

\section{In vitro antimicrobial screen}

501 For the initial staphylococci screen, single clone-derived cultures of animal-derived staphylococci were used as competitor isolates against the growth of methicillin-resistant $S$. pseudintermedius ST71. Each pure culture, including positive and negative control strains, were first streaked onto 3\% TSB agar plates and a single colony was transferred to $1 \mathrm{ml}$ of TSB in a deep 96 well plate (Thermo). The CoNS plate was sealed with sterile Aeraseal film (Sigma, St. Louis, MO) and cultured at $37^{\circ} \mathrm{C}$ overnight with shaking at $250 \mathrm{rpm}$. Bacterial growth was evaluated by measuring OD600 with only bacteria that grew to a density (OD600 > 6.0) used for subsequent analysis. To measure antimicrobial activity in the secreted supernatant, the animal-derived staphylococci supernatant from overnight cultures were harvested and centrifuged through several 96-well $0.2 \mu \mathrm{m}$ sterile filter plates (Corning). Next, $1 \times 10^{5} \mathrm{CFU}$ of S. pseudintermedius ST71 was inoculated into $150 \mu \mathrm{l}$ of $100 \%, 50 \%$ or $25 \%$ sterile supernatant supplemented with fresh $3 \%$ TSB and grown on a plate shaker for $18 \mathrm{~h}$ at $30^{\circ} \mathrm{C}$. To measure antimicrobial activity from the live agar co-culture assays, $20 \mu$ of overnight $S$. pseudintermedius ST71 culture was first inoculated into $45^{\circ} \mathrm{C}$ molten TSB and immobilized after pouring and cooling into square petri dishes with grids. Overnight cultures of animalderived staphylococci were centrifuged to pellet the bacteria, washed 2X with PBS and resuspended in fresh TSB. The culture $(10 \mu \mathrm{l})$ was inoculated onto a $13 \mathrm{~mm}$ grid of the $S$. pseudintermedius agar plates and cultured overnight at $30^{\circ} \mathrm{C}$. The resulting zones of inhibition from antimicrobial isolates were imaged using the camera feature on an iPhone 12.

\section{Extraction and purification of antimicrobials from bacterial supernatant}

Supernatant from overnight cultures of selected human and animal-derived staphylococci were first sterilized by filtration through a $0.22-\mu \mathrm{m}$ Millipore filter. Activity was precipitated by ammonium sulfate ( $75 \%$ saturation) for $1 \mathrm{~h}$, under constant rotation followed by centrifugation at 4,000 $\mathrm{x}$ g for $45 \mathrm{~min}$ and re-suspension of the pellet in $\mathrm{dH}_{2} \mathrm{O}$. To test stability, the precipitate was boiled at $95^{\circ} \mathrm{C}$ for 30 minutes or stored in a sterile eppendorf tube at room temperate for 1 week. Antimicrobial activity was measured by radial diffusion assay. Sterile supernatant of S. 
felis strains were subject to n-Butanol extraction and purification as previously reported (Joo and Otto, 2014). Briefly, in each tube $10 \mathrm{ml}$ of butanol was added to $30 \mathrm{ml}$ of supernatant and incubated at $37^{\circ} \mathrm{C}$ for $2 \mathrm{~h}$ under constant rotation. The tubes were then set aside for several mins until the butanol phase settled. After centrifugation at $2000 \mathrm{x}$ g for $5 \mathrm{~min}$, the upper butanol phase was collected and lyophilized in a SpeedVac vacuum concentrator. The lyophilized extract was resuspended and concentrated to $10 \mathrm{mg} / \mathrm{ml}$ in DMSO.

\section{Determination of minimum inhibitory concentration (MIC)}

MIC values were determined using a broth micro dilution method. Bacterial cells were grown to mid-late $\log$ phase, to an OD600 $\mathrm{nm}$ value of roughly 1.0 for each bacterial strain and then normalized to $1 \times 10^{7} \mathrm{CFU} / \mathrm{mL}$. The PSM peptides or butanol extracts were dissolved in DMSO to a stock concentration of $10 \mathrm{mg} / \mathrm{ml}$. The stock concentrations of antibiotics that were watersoluble were prepared with $\mathrm{H}_{2} \mathrm{O}$ or $100 \%$ ethanol if water-insoluble. The $1 \times 10^{7} \mathrm{CFU} / \mathrm{ml}$ bacterial cultures $(10 \mu \mathrm{l})$ were aliquoted into 96 -well microtiter plates and mixed with $95 \mu \mathrm{L}$ of media with or without 2-fold dilutions of the conditioned supernatant, PSM peptides, butanol extracts or antibiotics and incubated for $16-18 \mathrm{~h}$ at $30^{\circ} \mathrm{C}$ with shaking at $250 \mathrm{rpm}$. Growth inhibition was determined by measuring the OD600 $\mathrm{nm}$ readings of each well using a microplate reader (SpectraMax iD3, Molecular Devices). The MIC of each bacterial strain was determined by the lowest peptide concentration that inhibited more than $80 \%$ bacterial growth.

\section{Crystal Violet staining for biofilm disruption}

549 Overnight culture of $S$. pseudintermedius ST71 was diluted in fresh TSB to $1 \times 10^{7} \mathrm{CFU} / \mathrm{ml}$ by incubated at $37^{\circ} \mathrm{C}$ without shaking for $4 \mathrm{~h}$ to initiate biofilm formation. Next, the supernatants were removed by washing the plates three times with $200 \mu \mathrm{l}$ of $\mathrm{dH}_{2} \mathrm{O}$. Subsequently, $150 \mu \mathrm{l}$ of control, was added to the biofilm for periods between $2-24 \mathrm{~h}$. After incubation, the supernatant was gently removed, and the biofilm was washed three times with $\mathrm{dH}_{2} \mathrm{O}$ followed by air drying. Next, $150 \mu \mathrm{l}$ of $0.1 \%$ crystal violet $(\mathrm{CV})$ solution was added to all wells containing biofilm. After 20 mins of incubation with CV dye, the excess CV was removed and each well was washed twice with $\mathrm{dH}_{2} \mathrm{O}$. Fixed $\mathrm{CV}$ dye was released from the biofilm by $70 \%$ ethanol, and absorbance was measured at $595 \mathrm{~nm}$. 
562 First step HPLC purification was carried out with $1 \mathrm{mg}$ of $\mathrm{S}$. felis $\mathrm{C} 4$ supernatant loaded onto

563 a Capcell Pak® C8 column (5 mm, $300 \mathrm{~A}^{\circ}, 4.6 \mathrm{~mm} 250 \mathrm{~mm}$ ) (Shiseido, Tokyo, Japan) using

564 a linear acetonitrile gradient from $10 \%$ to $60 \%$ in $0.1 \%(\mathrm{v} / \mathrm{v})$ trifluoroacetic acid at a flow rate

565 of $1.0 \mathrm{ml} / \mathrm{min}$. The resulting fractions were lyophilized, then resuspended in water, and

566 antimicrobial activity assessed by liquid culture assay. Up to five sequential purifications were

567 carried out with each antimicrobial fraction pooled together for the second HPLC purification.

568 A linear gradient of acetonitrile from $25 \%$ to $50 \%$ was used for the second purification. PSM

569 peptides were synthesized with $\mathrm{N}$-terminal formulation to at least $95 \%$ purity by a commercial

570 vendor (LifeTein LLC, Somerset, NJ).

\section{Silver Stain and acetone precipitation of antimicrobial fractions}

573 Twenty $\mu \mathrm{g}$ of protein from sources including the antimicrobial HPLC fractions, butanol extracts and crude supernatant were loaded onto a Novex 16\% Tricine gel and subjected to SDS-PAGE. Silver staining and de-staining of the protein gels were performed according to the manufacturer's instructions (Thermo Pierce Silver Stain Kit). A previously published protocol for acetone extraction of AMPs from SDS gels was used (Burgess, 2009). Briefly, a sterile razor blade was used to excise gel slices according to protein size. The gel slices were cut into small pieces and immersed in $\mathrm{dH}_{2} \mathrm{O}$ for $4 \mathrm{~h}$, with regular vortexing to elute proteins. The eluted protein was mixed with four volumes of ice-cold acetone for $1 \mathrm{~h}$ at $-20^{\circ} \mathrm{C}$. The samples were centrifuged at $16,000 \mathrm{x}$ g for 15 mins at $4^{\circ} \mathrm{C}$. The supernatant was removed and lyophilized (acetone-soluble fraction) and the resulting pellet air dried briefly and resuspended in $\mathrm{dH}_{2} \mathrm{O}$ (acetone-insoluble fraction). The antimicrobial activity of both fractions was tested by radial diffusion agar assay against $S$. pseudintermedius ST71.

\section{Immunoblot}

587 NHEK cells were treated with Poly I:C $(0.4 \mu \mathrm{g} / \mathrm{ml})$, PSM $\beta 2(10 \mu \mathrm{g} / \mathrm{ml})$ or Poly I:C and PSM $\beta 2$ combined for $15 \mathrm{~min}, 30 \mathrm{~min}$ or $60 \mathrm{~min}$ and cells lysed in complete RIPA buffer supplemented with $1 \mathrm{X}$ protease and phosphatase inhibitor cocktail (Life Technologies, USA). The lysate was centrifuged at $4^{\circ} \mathrm{C}$, at $13,000 \mathrm{rpm}$ for $20 \mathrm{~min}$ and the total cytoplasmic supernatant fraction was kept at $-80{ }^{\circ} \mathrm{C}$, until future use. The total protein amount was quantified for each treatment using the Pierce ${ }^{\mathrm{TM}}$ BCA Protein Assay Kit according to manufacturer's instructions. Fifteen $\mu \mathrm{g}$ of total protein was loaded onto a 4-20\% Mini-PROTEAN TGX gel (Bio-Rad), then transferred to a polyvinylidene difluoride (PVDF) membrane and probed with the following primary antibodies: P-TBK1/NAK (D52C2), TBK1/NAK (D1B4), P-IRF-3 (S396), IRF-3 
596 (D83B9), COX IV (3E11). IRDye conjugated anti-rabbit and anti-mouse secondary antibodies

597 (IRDye800CW; Licor, USA) were used. The images were acquired on an Odyssey CLx 598 Imaging System (Licor, USA).

599

\section{Mass spectrometry}

601 Four fractions of interest (ranging from 10-20 $\mu \mathrm{g} / \mathrm{mL}$ ) were dried under vacuum and 602 resuspended in $15 \mu \mathrm{L}$ of $5 \%$ acetonitrile with $5 \%$ formic acid. Next, individual LC-MS 603 experiments were conducted on $6 \mu \mathrm{L}$ of each sample through 85 minutes of data acquisition on 604 an Orbitrap Fusion (Thermo Fisher Scientific) mass spectrometer with an in-line Easy-nLC 6051000 (Thermo Fisher Scientific). A home-pulled and packed $30 \mathrm{~cm}$ column was triple-packed 606 with $0.5 \mathrm{~cm}, 0.5 \mathrm{~cm}$ and $30 \mathrm{~cm}$ of $5 \mu \mathrm{m} \mathrm{C} 4,3 \mu \mathrm{m} \mathrm{C} 18$, and $1.8 \mu \mathrm{m} \mathrm{C} 18$ respectively and heated 607 to $60{ }^{\circ} \mathrm{C}$ for use as the analytical column. Peptides were first loaded at 500 bar which was 608 followed by a chromatography gradient ranging from 6 to $25 \%$ acetonitrile over 70 minutes 609 followed by a 5-minute gradient to $100 \%$ acetonitrile, which was held for 10 minutes. 610 Electrospray ionization was performed by applying $2000 \mathrm{~V}$ through a stainless-steel T-junction 611 connecting the analytical column and Easy-nLC system. Each sample was followed by four 612 washes starting with a gradient from 3 to $100 \%$ acetonitrile over 15 minutes with an additional 61310 minutes at $100 \%$ acetonitrile. An m/z range of 375-1500 was scanned for peptides with 614 charge states between 2-6. Centroided data was used for quantitation of peaks. Acquisition was 615 run in a data-dependent positive ion mode. Raw spectra was searched in Proteome Discoverer 616 Version 2.1 against 6-frame translated databases based of a uniprot reference database for 617 Staphylococcus felis ATCC 49168 (Uniprot proteome UP000243559, accessed 06/26/2019) as 618 well as in-house sequencing of S. felis C4. Data were searched using the Sequest algorithm 619 (Eng et al., 1994) using a reverse database approach to control peptide and protein false 620 discoveries to $1 \%$ (Elias and Gygi, 2007). No enzyme was specified in the search and a 621 minimum peptide length was set to 6 amino acids. Search parameters included a precursor mass 622 tolerance of $50 \mathrm{ppm}$ and fragment mass tolerance of $0.6 \mathrm{Da}$ and variable oxidation for 623 modifications.

626 DNA was extracted from $S$. felis $\mathrm{C} 4$ using the UltraClean ${ }^{\mathrm{TM}}$ Microbial DNA Isolation Kit 627 (MoBio) according to the manufacturer's instructions. The library was prepared using Nextera 628 DNA Flex library preparation kit according to the manufacturer's instructions (Illumina, San 629 Diego, CA). The library was diluted to $1.0 \mathrm{nM}$, then sequenced for 300 cycles using the Illumina 
630 NovaSeq system to generate $150 \mathrm{bp}$ paired-end reads with $794 \mathrm{x}$ coverage that was reduced to

631 100x coverage for read mapping. Fastq files from $S$. felis $\mathrm{C} 4$ were trimmed using Trimmomatic

632 (Bolger et al., 2014), then assembled using SPAdes Genome Assembler v.3.14.1. The S. felis

633 C4 genome was annotated using the RAST tool kit via the Pathosystems Resource Integration

634 Center (PATRIC) database (Wattam et al., 2014) and genes encoding PSM $\beta$ were identified

635 by BLASTn.

636

\section{ATP determination}

638 The intracellular ATP levels of $S$. pseudintermedius ST71 treated with S. felis C4 butanol 639 extract were measured following the manufacturer's instructions (ReadiUse ${ }^{\mathrm{TM}}$ Rapid 640 Luminometric ATP Assay Kit). Briefly, an overnight S. pseudintermedius ST71 culture was 641 sub-cultured to an OD of 0.5 at $37^{\circ} \mathrm{C}$. The bacteria were pelleted, washed with fresh TSB and 642 incubated with various concentrations $(0-320 \mu \mathrm{g} / \mathrm{ml})$ of $S$. felis $\mathrm{C} 4$ butanol extract for $1 \mathrm{~h}$. The 643 bacterial cultures were centrifuged at $10,000 \times \mathrm{g}$ for $5 \mathrm{~min}$ at $4{ }^{\circ} \mathrm{C}$. The bacterial pellet was 644 lysed by lysozyme and centrifuged. The bacterial supernatant was mixed with an equal volume 645 of detecting solution in a 96 well plate and incubated at room temperature for $20 \mathrm{~min}$. ATP 646 luminescence was read using a SpectraMax iD3 (Molecular Devices).

\section{Reactive Oxygen Species (ROS) measurement}

649 The levels of reactive oxygen species (ROS) in S. pseudintermedius ST71 that was treated with 650 S. felis $\mathrm{C} 4$ butanol extract were measured with $2^{\prime}, 7^{\prime}$-dichlorofluorescein diacetate (DCFDA) 651 following the manufacturer's instructions (DCFDA/H2DCFDA Abcam cellular ROS assay 652 kit). Briefly, an overnight culture of S. pseudintermedius ST71 was sub-cultured to an OD of 6530.5 at $37^{\circ} \mathrm{C}$. The bacteria were pelleted and re-suspended in fresh TSB. DCFDA was added to 654 a final concentration of $20 \mu \mathrm{M}$ to the bacterial culture incubated with various concentrations 655 of extract $(0-320 \mu \mathrm{g} / \mathrm{ml})$ at $37^{\circ} \mathrm{C}$ for 1 hour. Fluorescence intensity was immediately measured 656 at an excitation wavelength of $488 \mathrm{~nm}$ and an emission wavelength of $525 \mathrm{~nm}$ using a 657 SpectraMax iD3 (Molecular Devices).

\section{Bacterial viability assay}

660 Dead or damaged bacteria induced by $S$. felis $\mathrm{C} 4$ extract were evaluated using the 661 LIVE/DEAD® BacLight ${ }^{\mathrm{TM}}$ Bacterial Viability Kit (Invitrogen, catalogue no. L7007). An 662 overnight $S$. pseudintermedius ST71 culture was washed with fresh TSB and OD adjusted to 6630.5 under treatment with different concentrations of extract $(0,0.2,8$, and $32 \mu \mathrm{g} / \mathrm{ml})$. After 

of stained bacteria were obtained using a EVOS M5000 fluorescent microscope.

\section{Transmission electron microscopy}

670 S. pseudintermedius ST71 cell pellets were immersed in modified Karnovsky's fixative (2\% glutaraldehyde and 2\% paraformaldehyde in $0.10 \mathrm{M}$ sodium cacodylate buffer, $\mathrm{pH} 7.4$ ) for at least $4 \mathrm{~h}$ and further postfixed in $1 \%$ osmium tetroxide in $0.1 \mathrm{M}$ cacodylate buffer for $1 \mathrm{hr}$ on ice. The cells were stained all at once with $2 \%$ uranyl acetate for $1 \mathrm{hr}$ on ice, then dehydrated in a graded series of ethanol (50-100\%) while remaining on ice. The cells were washed with $100 \%$ ethanol and washed twice with acetone (10 min each) and embedded with Durcupan. Sections were cut at $60 \mathrm{~nm}$ on a Leica UCT ultramicrotome, and picked up on 300 mesh copper grids. Sections were post-stained with 2\% uranyl acetate for 5 minutes and Sato's lead stain for 1 minute. Grids were viewed using a JEOL JEM-1400Plus (JEOL, Peabody, MA) transmission electron microscope and photographed using a Gatan OneView 4K digital camera (Gatan, Pleasanton, CA).

\section{Mouse skin colonization and infection with $S$. pseudintermedius}

Mouse skin colonization. All experiments involving live animal work were performed in accordance with the approval of the University of California, San Diego Institutional Animal Care and Use Guidelines (protocol no. S09074). For mouse skin challenge experiments involving $S$. felis strains and S. pseudintermedius ST71, the dorsal skin of hairless age-matched 8-10 week-old SKH1 mice ( $\mathrm{n}=2$, per treatment) were scrubbed with alcohol wipes and $5 \times 10^{6} / \mathrm{cm}^{2}$ or $5 \times 10^{7} / \mathrm{cm}^{2}$ CFU of overnight cultured S. felis C4, S. felis ATCC 49168 or $S$. pseudintermedius ST71 was inoculated onto 1 x $1 \mathrm{~cm}$ sterile gauze pads, which were placed onto the dorsal skin and secured with wound dressing film (Tegaderm [3M]) film for $72 \mathrm{~h}$. For experiments involving live $S$. felis $\mathrm{C} 4$ bacteria or extract topical treatment, the dorsal skin of age-matched 8-10 week-old C57BL/6 mice ( $\mathrm{n}=4$, per treatment) was shaved and depilated by using Nair cream followed by removal with alcohol wipes. The skin was allowed to recover from hair removal for at least $24 \mathrm{~h}$ before the application of bacteria. Prior to bacterial challenge, the dorsal skin was tape-stripped and $S$ pseudintermedius ST71 agar disks (3\% tryptic soy broth [TSB], $2 \%$ agar; diameter $8 \mathrm{~mm}$ ) containing $5 \times 10^{7} \mathrm{CFU}$ was applied to the 
with Tegaderm and a bandage was applied to hold the agar disk or gauze in place for the duration of the treatment. The bandage, Tegaderm and agar disk were removed and $5 \times 10^{7} / \mathrm{cm}^{2}$ CFU of overnight cultured $S$. felis $\mathrm{C} 4$, or $150 \mu \mathrm{l}$ of extract $(10 \mathrm{mg} / \mathrm{ml})$ or $3 \%$ TSB control, was inoculated onto $2 \times 2 \mathrm{~cm}$ sterile gauze pads and applied to the infected site every $24 \mathrm{~h}$ for $72 \mathrm{~h}$. After the treatment of mouse skin with live bacteria or extract, the dressing film and gauze pad were removed, and surface bacteria were collected using a swab soaked in TSB-glycerol solution. The swab head was then placed in $1 \mathrm{~mL}$ of TSB-glycerol solution, vortexed (30 seconds), serial-diluted, and plated onto Baird Parker agar plates supplemented with egg yolk tellurite for enumeration of coagulase-positive staphylococci (Carter, 1960) or mannitol salt agar plates for enumeration of all surface staphylococci (Parisi and Hamory, 1986).

Mouse infection and dermonecrosis model. The day prior to bacterial infection, the dorsal skin of age-matched 8-10 week old C57BL/6 mice ( $\mathrm{n}=5$, per treatment) was shaved and depilated by using Nair cream followed by removal with alcohol wipes. A $50 \mu$ inoculum suspension containing $1 \times 10^{7} \mathrm{CFU}$ of late log phase $S$. pseudintermedius ST71 in PBS was intradermally injected into the dorsal skin using $0.3 \mathrm{~mL} / 31$-gauge insulin syringe (BD, Franklin Lakes, NJ). At $1 \mathrm{~h}$ post-infection, a $50 \mu \mathrm{l}$ suspension of the S. felis $\mathrm{C} 4$ extract $(250 \mu \mathrm{g}$, at a concentration of $5 \mathrm{mg} / \mathrm{ml}$ in $25 \% \mathrm{DMSO}$ ) or a control suspension of $1 \mathrm{X}$ PBS in $25 \% \mathrm{DMSO}$ was injected twice in two separate skin sites directly adjacent to the bacterial injection site. Body weights of the mice were measured before and after infection every day for 14 days. To determine lesion size, a ruler was positioned adjacent to the mouse skin lesions and digital photos were taken daily with a Kodak PIXPRO Astro Zoom AZ421 and analyzed via ImageJ software (National Institutes of Health Research Services Branch, Bethesda, MD, USA). Lesion size in $\mathrm{mm}^{2}$ was measured by calculating the length $\mathrm{x}$ width.

\section{Quantitative real-time PCR}

mRNA transcript abundance was measured by qPCR in NHEKs stimulated with or without TLR2/6 agonist MALP-2 $(200 \mathrm{ng} / \mathrm{ml})$ or TLR3 agonist Poly I:C $(0.4 \mu \mathrm{g} / \mathrm{ml})$ in the presence or absence of $S$. felis C4 extract, PSM $\beta 2$ or PSM $\beta 3(10 \mu \mathrm{g} / \mathrm{ml})$ or DMSO control $(0.1 \%)$ at $4 \mathrm{~h}$ post-treatment. RNA was extracted from NHEK cells using Pure Link RNA isolation kit (Life Technologies, USA) according to manufacturer's instructions. RNA was quantified on a Nanodrop 2000/200c spectrophotometer (Thermo Fisher, USA). Purified RNA (500 $\mu$ g) was used to synthesize cDNA using the iScript ${ }^{\mathrm{TM}}$ cDNA Synthesis Kit (Bio-Rad, USA). Pre 
731 Developed Taqman ${ }^{\circledR}$ (Thermo Fisher, USA) and SYBR-Green gene expression assays (Integrated DNA Technologies, USA) were used to evaluate mRNA transcript levels.

\section{RNA Sequencing}

NHEK cells were treated with DMSO (0.1\%) control, PSM $32(10 \mu \mathrm{g} / \mathrm{ml})$, Poly I:C $(0.4 \mu \mathrm{g} / \mathrm{ml})$ or PSM $\beta 2$ and Poly I:C combined, all in triplicate wells, for $4 \mathrm{~h}$ or $24 \mathrm{~h}$ in and RNA was extracted using the PureLink RNA mini kit and triplicate samples were pooled together for each treatment. Isolated RNA was submitted to the UCSD IGM Genomics Center for RNAsequencing performed on a high-output run V4 platform (Illumina, USA) with a single read 100 cycle runs. Data alignment was performed using Partek ${ }^{\circledR}$ Flow ${ }^{\circledR}$ genomic analysis software (Partek, USA) with Tophat2 (version 2.0.8) Gene ontology (GO) enrichment analysis was performed on differentially regulated genes ( $\geq 1.5$-fold) using DAVID 6.8 .

\section{Statistical analysis}

745 Significant differences between the means of the different treatments were evaluated using 746 GraphPad Prism version 7.03 (GraphPad Software, Inc., La Jolla, CA). Either unpaired, twotailed Student's $t$ test or one-way analysis of variance (ANOVA) followed by Dunnett's or Turkey's multiple comparisons test were used for statistical analysis and indicated in the respective figure legends. Differences were considered statistically significant with a $p$ value of $<0.05$.

\section{ACKNOWLEDGEMENTS}

The authors would like to acknowledge the participants for their assistance in this project. We thank Ying Jones of the UCSD/CMM electron microscopy facility for TEM sample preparation and Timothy Meerloo for imaging assistance. The EM facility is supported by NIH equipment grant 1S10OD023527. We thank Nina J. Gao for providing strains. We thank Jamie Boehmer for her very helpful edits. We also thank Gayathri Kalla, Joe Pogliano, Kit Pogliano and Gemma Ma.

\section{FUNDING}

761 This publication includes data generated at the UC San Diego IGM Genomics Center utilizing an Illumina NovaSeq 6000 that was purchased with funding from a National Institutes of Health SIG grant (\#S10 OD026929). R.H.M. was supported through a UCSD training grant from the NIH/NIDDK Gastroenterology Training Program (T32 DK007202). 


\section{CONFLICT OF INTEREST}

767 R.L.G. is a co-founder, scientific advisor, consultant and has equity in MatriSys Biosciences

768 and is a consultant, receives income and has equity in Sente. K.W. and R.L.G are co-

769 inventors of technology described in this report that has been disclosed to the University of

770 California San Diego.

\section{SUPPLEMENTARY FIGURES}

A

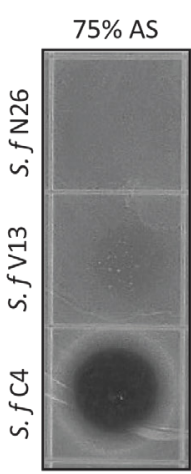

B
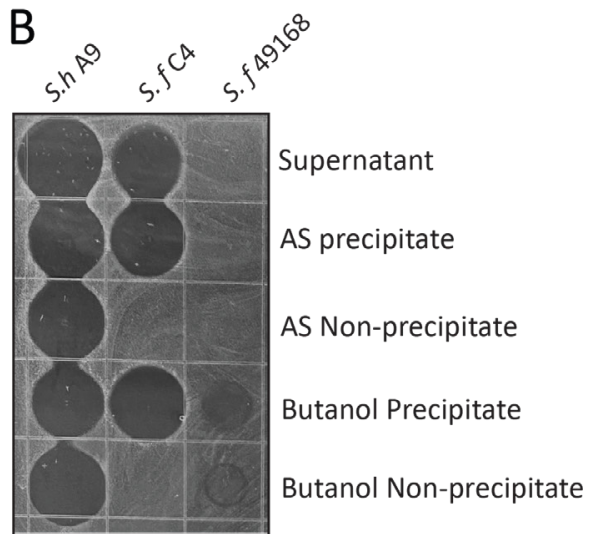
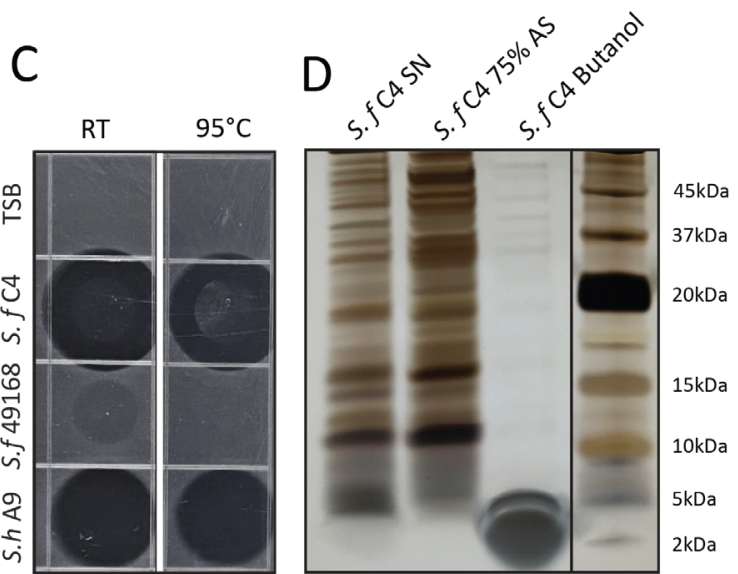

775

Suppl. Figure 2. Generation of a partially purified antimicrobial extract from S. felis $\mathbf{C 4}$.

776

(A) Supernatant of indicated antimicrobial S. felis strains (S. $f \mathrm{C} 4, \mathrm{~N} 26, \mathrm{~V} 13)$ were incubated with 75\% ammonium sulfate (AS) and the resulting precipitate was inoculated onto agar containing $S$. pseudintermedius ST71, to determine antimicrobial activity. (B) Supernatant of S. felis C4, positivecontrol S. hominis A9, or negative-control $S$. felis ATCC 49168 was extracted in $75 \%$ AS or $25 \%$ nbutanol and activity of the precipitate and non-precipitate fractions were assayed against $S$. pseudintermedius ST71. (C) Supernatant of S. felis C4 (S. fC4), S. hominis A9 (S. h A9), S. capitis E12

782 (S. c E12) or TSB alone, were maintained at room temperature (RT) for 1 week or boiled at $95^{\circ} \mathrm{C}$, for

78330 mins and activity determined by inoculation directly onto agar containing $S$. pseudintermedius ST71.

784 (D) Total protein silver stain of S. felis C4 supernatant before precipitation or after precipitation in $75 \%$ AS or $25 \%$ butanol. 
A

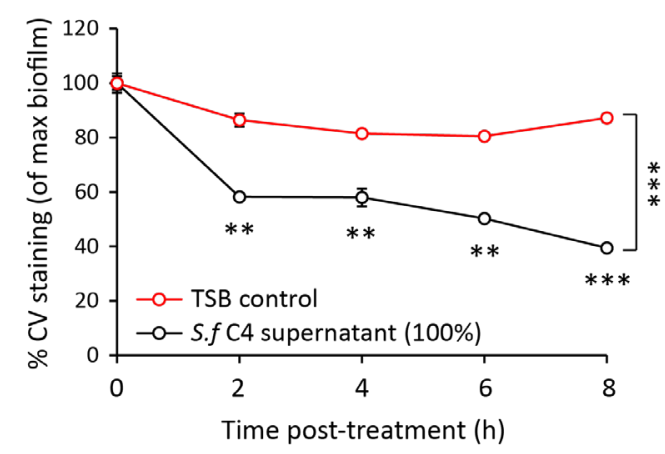

B

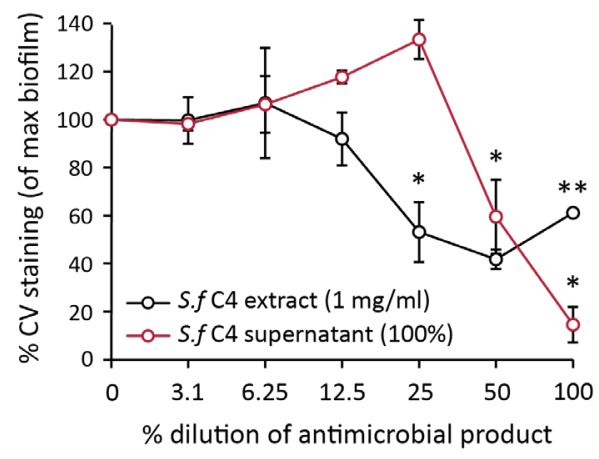

$\%$ dilution of antimicrobial product

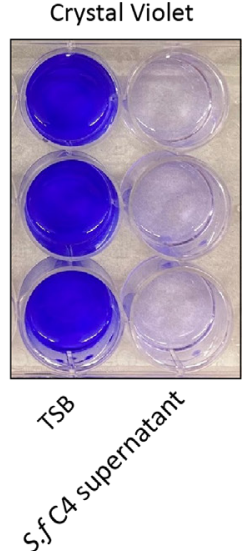

Suppl. Figure 2. S. felis $\mathrm{C} 4$ supernatant and extract disrupt $S$. pseudintermedius biofilm.

(A) Decrease in crystal violet staining of a $4 \mathrm{~h}$ preformed $S$. pseudintermedius ST71 biofilm during $8 \mathrm{~h}$ incubation with $100 \%$ S. felis C4 sterile supernatant. (B) Decrease in crystal violet staining of a $4 \mathrm{~h}$ preformed S. pseudintermedius ST71 biofilm after $24 \mathrm{~h}$ incubation with serially diluted S. felis $\mathrm{C} 4$ sterile supernatant (100\%) or S. felis C4 extract $(1 \mathrm{mg} / \mathrm{ml})$. Right inset is a representative image of crystal

793 violet staining after incubation with $100 \%$ S. felis C4 supernatant. (A-B) Error bars indicate SEM.

794 Representative of two separate experiments. A two-tailed, unpaired Student's t test was performed. $p$ values: ${ }^{*} p<0.05 ; * * p<0.01 ; * * * p<0.001$. 


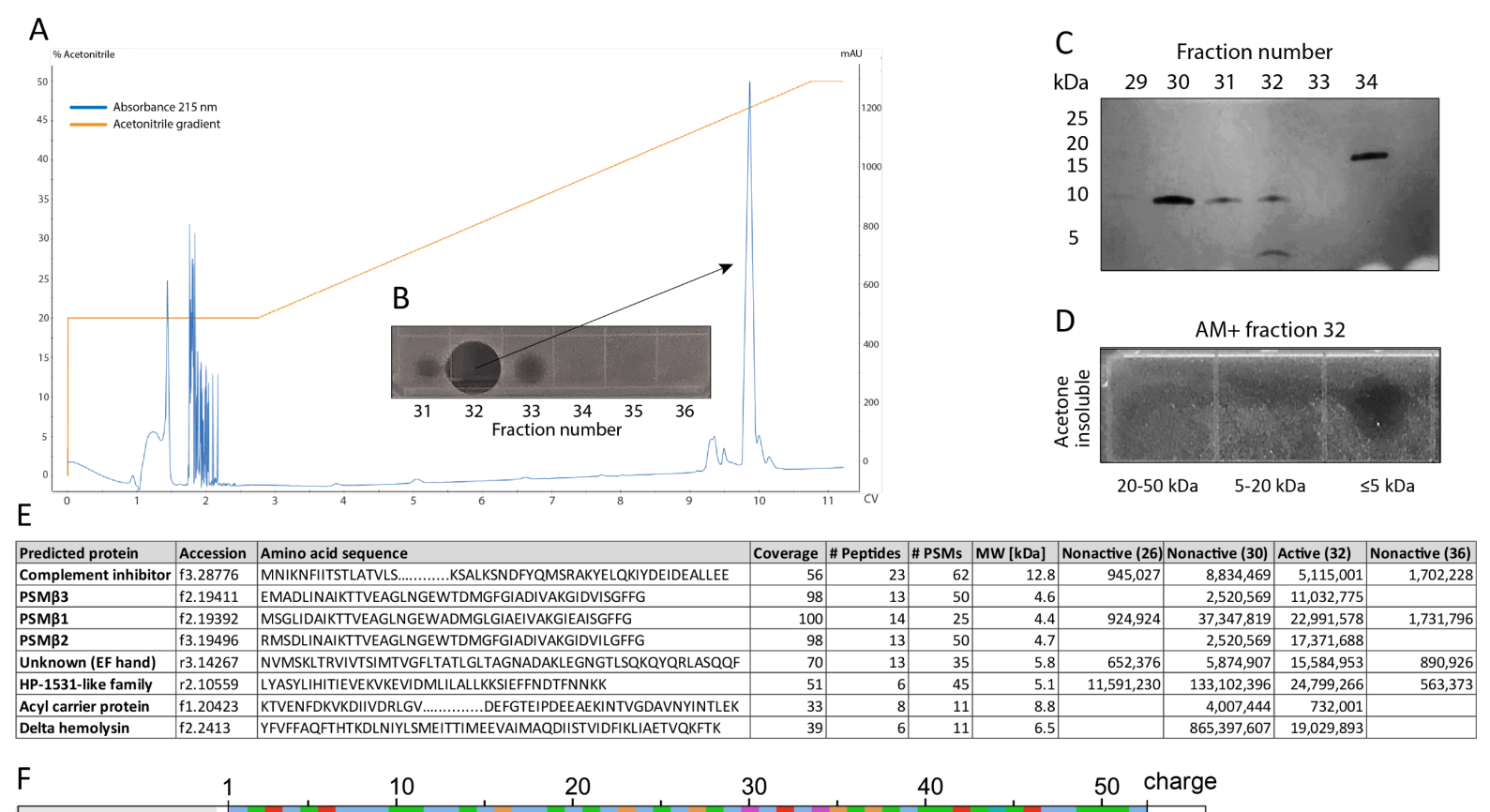

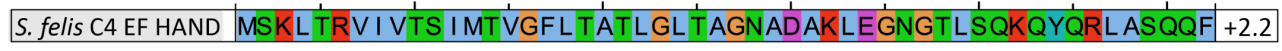

G

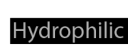

Hydrophobic
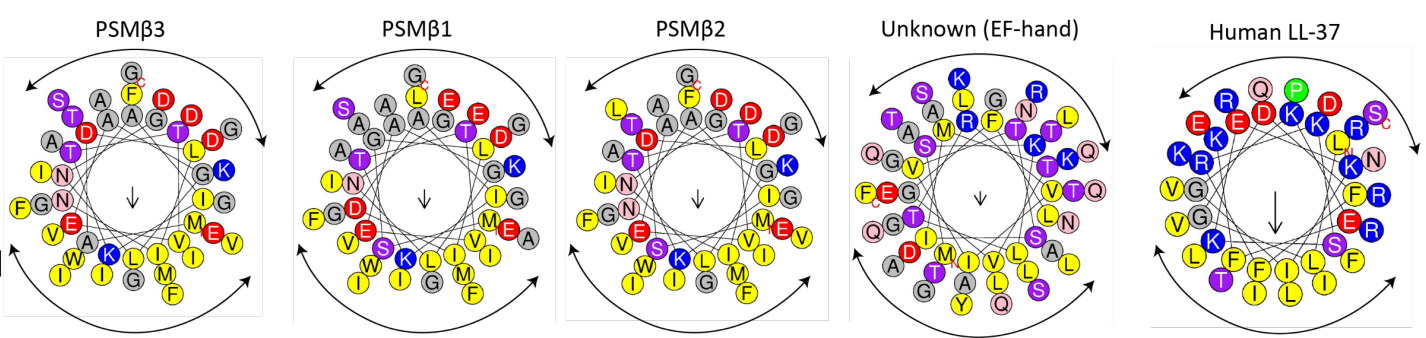

Suppl. Figure 3. HPLC purification yields antimicrobial fraction from S. felis $\mathrm{C} 4$ supernatant

(A) Reverse-phase high-performance liquid chromatography (HPLC) elution profile from sterile supernatant of S. felis $\mathrm{C} 4$ strain loaded onto a C8 column. (B) Inset image of antimicrobial activity exhibited by fraction 32 against S. pseudintermedius ST71 corresponding to the indicated peak. (C) Silver stain of total protein content in the different fractions indicated. (D) Radial diffusion assay of antimicrobial activity of the AM+ fraction 32 after extraction and acetone precipitation of proteins within different sized silver stain gel fragments. (E) Mass spectrometry (MS) table of the top 8 peptide hits obtained from HPLC fractions that were active (fraction 32) or inactive $(26,30,36)$ against $S$. pseudintermedius ST71. (F) ClustalW multiple amino acid sequence alignment of all three S. felis C4 genetically-encoded PSM $\beta$ peptides with predicted net charge at pH 7.4 (Prot pi) and amino acid sequence of a EF-hand domain-containing peptide with unknown function. (G) Alpha helical wheel plots of S. felis C4 PSM $\beta 1$-3, EF-hand domain peptide and human LL-37 peptide, indicating conserved $\alpha$-helical, amphipathic-like structures with indicated hydrophobic yellow residues confined to one side (indicated by arrow) and grey hydrophilic residues on the opposing side. 

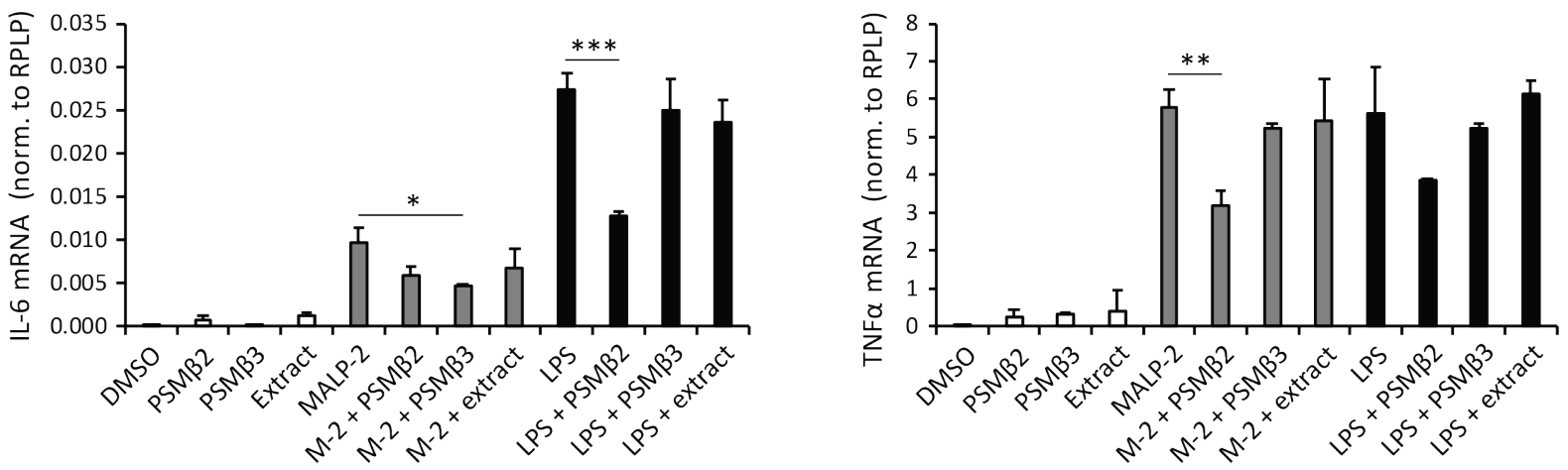

Transcript abundance of inflammatory cytokines IL-6 and TNF $\alpha$ in THP-1 cells stimulated with or without TLR2/6 agonist MALP-2 $(200 \mathrm{ng} / \mathrm{ml})$ or TLR4 agonist LPS $(1 \mu \mathrm{g} / \mathrm{ml})$ in the presence or absence of $S$. felis C4 extract, PSM $\beta 2$ or PSM $\beta 3(10 \mu \mathrm{g} / \mathrm{ml})$ or DMSO control $(0.1 \%) 4$ h post-treatment. Error bars indicate SEM. One-way ANOVA with multiple corrections (Turkey correction) was performed. $p$ values: ${ }^{*} \mathrm{p}<0.05 ;{ }^{* *}, \mathrm{p}<0.01 ; * * * \mathrm{p}<0.001$.

821

822

823

824

825

826

827

828

829

830
A

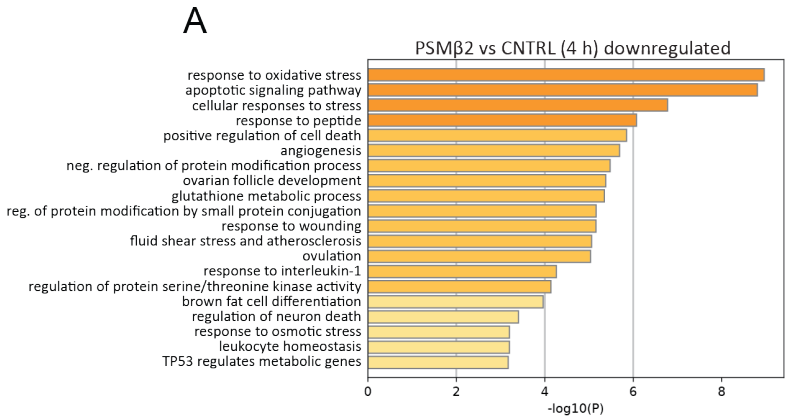

B

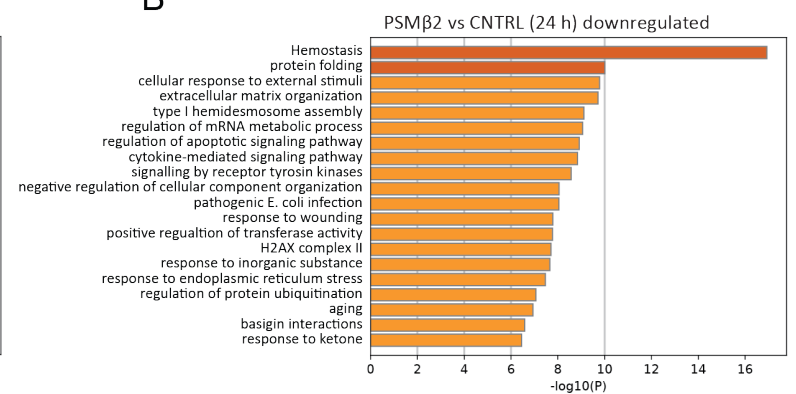

Suppl. Figure 5. PSM $\beta 2$ supresses TLR3-mediated cellular activation and downregulates responses to bacterial pathogens.

(A-B) GO pathway analysis of genes down-regulated in NHEKs at $4 \mathrm{~h}$ (A) and $24 \mathrm{~h}$ (B) after treatment with PSM 32 versus DMSO control.

\section{REFERENCES}

Banovic F, Olivry T, Bäumer W, Paps J, Stahl J, Rogers A, Jacob M. 2018. Diluted sodium hypochlorite (bleach) in dogs: antiseptic efficacy, local tolerability and in vitro effect on skin barrier function and inflammation. Vet Dermatol 29:5-6. doi:10.1111/vde.12487 Bolger AM, Lohse M, Usadel B. 2014. Trimmomatic: A flexible trimmer for Illumina sequence data. Bioinformatics 30:2114-2120. doi:10.1093/bioinformatics/btu170 Börjesson S, Gómez-Sanz E, Ekström K, Torres C, Grönlund U. 2015. Staphylococcus pseudintermedius can be misdiagnosed as Staphylococcus aureus in humans with dog 
bite wounds. Eur J Clin Microbiol Infect Dis 34:839-844. doi:10.1007/s10096-014$2300-\mathrm{y}$

Botelho D, Wall MJ, Vieira DB, Fitzsimmons S, Liu F, Doucette A. 2010. Top-down and bottom-up proteomics of sds-containing solutions following mass-based separation. $J$ Proteome Res 9:2863-2870. doi:10.1021/pr900949p

Bradley CW, Morris DO, Rankin SC, Cain CL, Misic AM, Houser T, Mauldin EA, Grice EA. 2016. Longitudinal evaluation of the skin microbiome and association with microenvironment and treatment in canine atopic dermatitis. $J$ Invest Dermatol 136:1182-1190. doi:10.1016/j.jid.2016.01.023

Burgess RR. 2009. Chapter 32 Elution of proteins from gelsmethods in enzymology. Academic Press Inc. pp. 565-572. doi:10.1016/S0076-6879(09)63032-9

Carter CH. 1960. Egg yolk agar for isolation of coagulase-positive staphylococci. J Bacteriol 79.

Chazin WJ. 2011. Relating form and function of EF-hand calcium binding proteins. Acc Chem Res 44:171-179. doi:10.1021/ar100110d

Cheung GYC, Joo HS, Chatterjee SS, Otto M. 2014. Phenol-soluble modulins - critical determinants of staphylococcal virulence. FEMS Microbiol Rev. doi:10.1111/15746976.12057

Chopra R, Vakharia PP, Sacotte R, Silverberg JI. 2017. Efficacy of bleach baths in reducing severity of atopic dermatitis: A systematic review and meta-analysis. Ann Allergy, Asthma Immunol 119:435-440. doi:10.1016/j.anai.2017.08.289

Claesen J, Spagnolo JB, Ramos SF, Kurita KL, Byrd AL, Aksenov AA, Melnik A V., Wong WR, Wang S, Hernandez RD, Donia MS, Dorrestein PC, Kong HH, Segre JA, Linington RG, Fischbach MA, Lemon KP. 2020. A Cutibacterium acnes antibiotic modulates human skin microbiota composition in hair follicles. Sci Transl Med 12. doi:10.1126/scitranslmed.aay5445

Cogen AL, Yamasaki K, Muto J, Sanchez KM, Alexander LC, Tanios J, Lai Y, Kim JE, Nizet V, Gallo RL. 2010. Staphylococcus epidermidis antimicrobial $\delta$-toxin (phenolsoluble modulin- $\gamma$ ) cooperates with host antimicrobial peptides to kill group A Streptococcus. PLoS One 5. doi:10.1371/journal.pone.0008557

Da F, Joo H-S, Cheung GYC, Villaruz AE, Rohde H, Luo X, Otto M. 2017. Phenol-soluble modulin toxins of Staphylococcus haemolyticus. Front Cell Infect Microbiol 7:206. doi:10.3389/fcimb.2017.00206

Darlow CA, Paidakakos N, Sikander M, Atkins B. 2017. A spinal infection with 
Staphylococcus pseudintermedius. BMJ Case Rep 2017. doi:10.1136/bcr-2017-221260

Di Domenico EG, Cavallo I, Bordignon V, Prignano G, Sperduti I, Gurtner A, Trento E, Toma L, Pimpinelli F, Capitanio B, Ensoli F. 2018. Inflammatory cytokines and biofilm production sustain Staphylococcus aureus outgrowth and persistence: A pivotal interplay in the pathogenesis of Atopic Dermatitis. Sci Rep 8. doi:10.1038/s41598-01827421-1

Duong AC, Cheung GYC, Otto M. 2012. Interaction of phenol-soluble modulins with phosphatidylcholine vesicles. Pathogens 1:3-11. doi:10.3390/pathogens1010003

Elias JE, Gygi SP. 2007. Target-decoy search strategy for increased confidence in large-scale protein identifications by mass spectrometry. Nat Methods 4:207-214. doi:10.1038/nmeth1019

Eng JK, McCormack AL, Yates JR. 1994. An approach to correlate tandem mass spectral data of peptides with amino acid sequences in a protein database. J Am Soc Mass Spectrom 5:976-989. doi:10.1016/1044-0305(94)80016-2

Fazakerley J, Nuttall T, Sales D, Schmidt V, Carter SD, Hart CA, McEwan NA. 2009. Staphylococcal colonization of mucosal and lesional skin sites in atopic and healthy dogs. Vet Dermatol 20:179-184. doi:10.1111/j.1365-3164.2009.00745.x

Ference EH, Danielian A, Kim HW, Yoo F, Kuan EC, Suh JD. 2019. Zoonotic Staphylococcus pseudintermedius sinonasal infections: risk factors and resistance patterns. Int Forum Allergy Rhinol 9:724-729. doi:10.1002/alr.22329

Frana TS, Beahm AR, Hanson BM, Kinyon JM, Layman LL, Karriker LA, Ramirez A, Smith TC. 2013. Isolation and characterization of methicillin-resistant Staphylococcus aureus from pork farms and visiting veterinary students. PLoS One 8: 53738. doi:10.1371/journal.pone.0053738

Garbacz K, Zarnowska S, Piechowicz L, Haras K. 2013. Pathogenicity potential of Staphylococcus pseudintermedius strains isolated from canine carriers and from dogs with infection signs. Virulence. doi:10.4161/viru.23526

Grice EA, Segre JA. 2011. The skin microbiome. Nat Rev Microbiol 9:244. doi:10.1038/nrmicro2537

Gueniche A, Knaudt B, Schuck E, Volz T, Bastien P, Martin R, Röcken M, Breton L, Biedermann T. 2008. Effects of nonpathogenic gram-negative bacterium Vitreoscilla filiformis lysate on atopic dermatitis: A prospective, randomized, double-blind, placebocontrolled clinical study. Br J Dermatol 159:1357-1363. doi:10.1111/j.1365- 
905

906

907

908

909

910

911

912

913

914

915

916

917

918

919

920

921

922

923

924

925

926

927

928

929

930

931

932

933

934

935

936

937

938

Joo HS, Cheung GYC, Otto M. 2011. Antimicrobial activity of community-associated methicillin-resistant Staphylococcus aureus is caused by phenol-soluble modulin derivatives. J Biol Chem 286:8933-8940. doi:10.1074/jbc.M111.221382

Joo HS, Otto M. 2014. The isolation and analysis of phenol-soluble modulins of Staphylococcus epidermidis. Methods Mol Biol 1106:93-100. doi:10.1007/978-1-62703736-5_7

Kong HH, Oh J, Deming C, Conlan S, Grice EA, Beatson MA, Nomicos E, Polley EC, Komarow HD, Mullikin J, Thomas J, Blakesley R, Young A, Chu G, Ramsahoye C, Lovett S, Han J, Legaspi R, Sison C, Montemayor C, Gregory M, Hargrove A, Johnson T, Riebow N, Schmidt B, Novotny B, Gupta J, Benjamin B, Brooks S, Coleman H, Ho SL, Schandler K, Stantripop M, Maduro Q, Bouffard G, Dekhtyar M, Guan X, Masiello C, Maskeri B, McDowell J, Park M, Vemulapalli M, Murray PR, Turner ML, Segre JA. 2012. Temporal shifts in the skin microbiome associated with disease flares and treatment in children with atopic dermatitis. Genome Res 22:850-859. doi:10.1101/gr.131029.111

Kumar R, Jangir PK, Das J, Taneja B, Sharma R. 2017. Genome analysis of Staphylococcus capitis TE8 reveals repertoire of antimicrobial peptides and adaptation strategies for growth on human skin. Sci Rep 7. doi:10.1038/s41598-017-11020-7

Kwiecinski J, Kahlmeter G, Jin T. 2015. Biofilm formation by staphylococcus aureus isolates from skin and soft tissue infections. Curr Microbiol 70:698-703. doi:10.1007/s00284014-0770-x

Lai PS, Allen JG, Hutchinson DS, Ajami NJ, Petrosino JF, Winters T, Hug C, Wartenberg GR, Vallarino J, Christiani DC. 2017. Impact of environmental microbiota on human microbiota of workers in academic mouse research facilities: An observational study. PLoS One 12:e0180969. doi:10.1371/journal.pone.0180969

Ma GC, Worthing KA, Ward MP, Norris JM. 2020. Commensal Staphylococci Including Methicillin-resistant Staphylococcus aureus from dogs and cats in remote New South Wales, Australia. Microb Ecol 79:164-174. doi:10.1007/s00248-019-01382-y

Mandhane PJ, Sears MR, Poulton R, Greene JM, Lou WYW, Taylor DR, Hancox RJ. 2009. Cats and dogs and the risk of atopy in childhood and adulthood. $J$ Allergy Clin Immunol 124:745-750.e4. doi:10.1016/j.jaci.2009.06.038

Marsella R, Girolomoni G. 2009. Canine models of atopic dermatitis: A useful tool with untapped potential. J Invest Dermatol. doi:10.1038/jid.2009.98

Myles IA, Williams KW, Reckhow JD, Jammeh ML, Pincus NB, Sastalla I, Saleem D, Stone 
KD, Datta SK. 2019. Transplantation of human skin microbiota in models of atopic dermatitis. JCI Insight 1. doi:10.1172/jci.insight.86955

Nakamura Y, Oscherwitz J, Cease KB, Chan SM, Muñoz-Planillo R, Hasegawa M, Villaruz

503:397-401. doi:10.1038/nature12655

Nakatsuji T, Chen TH, Butcher AM, Trzoss LL, Nam SJ, Shirakawa KT, Zhou W, Oh J, Otto

Nakatsuji T, Chen TH, Narala S, Chun KA, Two AM, Yun T, Shafiq F, Kotol PF, doi:10.1126/scitranslmed.aah4680

Nakatsuji T, Chen TH, Two AM, Chun KA, Narala S, Geha RS, Hata TR, Gallo RL. 2016. cytokine expression. J Invest Dermatol. doi:10.1016/j.jid.2016.05.127

Nakatsuji T, Gallo RL. 2019. The role of the skin microbiome in atopic dermatitis. Ann Allergy, Asthma Immunol. doi:10.1016/j.anai.2018.12.003

Nakatsuji T, Gallo RL. 2012. Antimicrobial peptides: Old molecules with new ideas. J Invest Dermatol. doi:10.1038/jid.2011.387

O’Neill AMAM, Nakatsuji T, Hayachi A, Williams MRMR, Mills RHRH, Gonzalez DJDJ, Gallo RLRL. 2020. Identification of a human skin commensal bacterium that selectively kills Cutibacterium acnes. J Invest Dermatol 140:1619-1628.e2. doi:10.1016/j.jid.2019.12.026

Older CE, Hoffmann AR, Hoover K, Banovic F. 2020. Characterization of cutaneous bacterial microbiota from superficial pyoderma forms in atopic dogs. Pathogens 9:1-12. doi:10.3390/pathogens 9080638

Otto M. 2009. Staphylococcus epidermidis - The "accidental" pathogen. Nat Rev Microbiol.

970 Parisi JT, Hamory BH. 1986. Simplified method for the isolation, identification, and characterization of Staphylococcus epidermidis in epidemiologic studies. Diagn Microbiol Infect Dis 4:29-35. doi:10.1016/0732-8893(86)90053-2 
Perreten V, Kadlec K, Schwarz S, Andersson UG, Finn M, Greko C, Moodley A, Kania SA, Frank LA, Bemis DA, Franco A, Iurescia M, Battisti A, Duim B, Wagenaar JA, van Duijkeren E, Weese JS, Fitzgerald JR, Rossano A, Guardabassi L. 2010. Clonal spread of methicillin-resistant Staphylococcus pseudintermedius in Europe and North America: An international multicentre study. J Antimicrob Chemother 65:1145-1154. doi:10.1093/jac/dkq078

Riegel P, Jesel-Morel L, Laventie B, Boisset S, Vandenesch F, Prévost G. 2011. Coagulasepositive Staphylococcus pseudintermedius from animals causing human endocarditis. Int J Med Microbiol 301:237-239. doi:10.1016/j.ijmm.2010.09.001

Robb AR, Wright ED, Foster AME, Walker R, Malone C. 2017. Skin infection caused by a novel strain of Staphylococcus pseudintermedius in a Siberian husky dog owner. JMM Case Reports 4. doi:10.1099/jmmcr.0.005087

Ross AA, Müller KM, Scott Weese J, Neufeld JD. 2018. Comprehensive skin microbiome analysis reveals the uniqueness of human skin and evidence for phylosymbiosis within the class Mammalia. Proc Natl Acad Sci U S A 115:E5786-E5795.

$$
\text { doi:10.1073/pnas.1801302115 }
$$

Ross AC, Vederas JC. 2011. Fundamental functionality: Recent developments in understanding the structure-activity relationships of lantibiotic peptides. J Antibiot (Tokyo). doi:10.1038/ja.2010.136

Sanford JA, Gallo RL. 2013. Functions of the skin microbiota in health and disease. Semin Immunol. doi:10.1016/j.smim.2013.09.005

Sawada Y, Tong Y, Barangi M, Hata T, Williams MR, Nakatsuji T, Gallo RL. 2019. Dilute bleach baths used for treatment of atopic dermatitis are not antimicrobial in vitro. $J$ Allergy Clin Immunol. doi:10.1016/j.jaci.2019.01.009

Silverberg JI. 2019. Comorbidities and the impact of atopic dermatitis. Ann Allergy, Asthma Immunol 123:144-151. doi:10.1016/j.anai.2019.04.020

Singh A, Walker M, Rousseau J, Weese JS. 2013. Characterization of the biofilm forming ability of Staphylococcus pseudintermedius from dogs. BMC Vet Res 9. doi:10.1186/1746-6148-9-93

Somayaji R, Priyantha MAR, Rubin JE, Church D. 2016. Human infections due to Staphylococcus pseudintermedius, an emerging zoonosis of canine origin: report of 24 cases. Diagn Microbiol Infect Dis 85:471-476. doi:10.1016/j.diagmicrobio.2016.05.008 Song J, Lauber C, Costello EK, Lozupone CA, Humphrey G, Berg-Lyons D, Caporaso JG, Knights D, Clemente JC, Nakielny S, Gordon JI, Fierer N, Knight R. 2013. Cohabiting 
family members share microbiota with one another and with their dogs. elife.elifesciences.org Song al eLife 2:458. doi:10.7554/eLife.00458

Song M, Liu Y, Huang X, Ding S, Wang Y, Shen J, Zhu K. 2020. A broad-spectrum antibiotic adjuvant reverses multidrug-resistant Gram-negative pathogens. Nat Microbiol 5:1040-1050. doi:10.1038/s41564-020-0723-z

Stegmann R, Burnens A, Maranta CA, Perreten V. 2010. Human infection associated with methicillin-resistant Staphylococcus pseudintermedius ST71. J Antimicrob Chemother 65:2047-2048. doi:10.1093/jac/dkq241

Walsh CT. 2008. The chemical versatility of natural-product assembly lines. Acc Chem Res 41:4-10. doi:10.1021/ar7000414

Wang R, Braughton KR, Kretschmer D, Bach THL, Queck SY, Li M, Kennedy AD, Dorward cytolytic peptides as key virulence determinants for community-associated MRSA. Nat Med 13:1510-1514. doi:10.1038/nm1656

Wattam AR, Abraham D, Dalay O, Disz TL, Driscoll T, Gabbard JL, Gillespie JJ, Gough R, Hix D, Kenyon R, MacHi D, Mao C, Nordberg EK, Olson R, Overbeek R, Pusch GD, Shukla M, Schulman J, Stevens RL, Sullivan DE, Vonstein V, Warren A, Will R, Wilson MJC, Yoo HS, Zhang C, Zhang Y, Sobral BW. 2014. PATRIC, the bacterial bioinformatics database and analysis resource. Nucleic Acids Res 42:D581. doi:10.1093/nar/gkt1099

Weese JS, Poma R, James F, Buenviaje G, Foster R, Slavic D. 2009. Case report. Can Vet J 50:655-656.

Williams MR, Costa SK, Zaramela LS, Khalil S, Todd DA, Winter HL, Sanford JA, O’Neill AM, Liggins MC, Nakatsuji T, Cech NB, Cheung AL, Zengler K, Horswill AR, Gallo RL. 2019. Quorum sensing between bacterial species on the skin protects against epidermal injury in atopic dermatitis. Sci Transl Med. doi:10.1126/scitranslmed.aat8329

Worthing K, Pang S, Trott DJ, Abraham S, Coombs GW, Jordan D, McIntyre L, Davies MR, Norris J. 2018. Characterisation of Staphylococcus felis isolated from cats using whole genome sequencing. Vet Microbiol 222:98-104. doi:10.1016/j.vetmic.2018.07.002

Worthing KA, Abraham S, Coombs GW, Pang S, Saputra S, Jordan D, Trott DJ, Norris JM. 2018. Clonal diversity and geographic distribution of methicillin-resistant Staphylococcus pseudintermedius from Australian animals: Discovery of novel sequence types. Vet Microbiol 213:58-65. doi:10.1016/j.vetmic.2017.11.018 Zeng P, Xu C, Cheng Q, Liu J, Gao W, Yang X, Wong KY, Chen S, Chan KF. 2019. Phenol- 
soluble-modulin-inspired amphipathic peptides have bactericidal activity against multidrug-resistant bacteria. ChemMedChem 14:1547-1559. doi:10.1002/cmdc.201900364

1044 Zhang Y, Bottinelli D, Lisacek F, Luban J, Strambio-De-Castillia C, Varesio E, Hopfgartner G. 2015. Optimization of human dendritic cell sample preparation for mass spectrometry-based proteomic studies. Anal Biochem 484:40-50. doi:10.1016/j.ab.2015.05.007

1048 Zipperer A, Konnerth MC, Laux C, Berscheid A, Janek D, Weidenmaier C, Burian M,

1049 Schilling NA, Slavetinsky C, Marschal M, Willmann M, Kalbacher H, Schittek B,

1050 Brötz-Oesterhelt H, Grond S, Peschel A, Krismer B. 2016. Human commensals

1051 producing a novel antibiotic impair pathogen colonization. Nature 535:511-516.

1052 doi:10.1038/nature 18634

1053

1054 